In: Journal of Logic, Language and Information 22 (2013), 195-230.

\title{
Paraconsistency and Plausible Argumentation in Generative Grammar: A Case Study
}

\author{
András Kertész and Csilla Rákosi
}

\begin{abstract}
While the analytical philosophy of science regards inconsistent theories as disastrous, Chomsky allows for the temporary tolerance of inconsistency between the hypotheses and the data. However, in linguistics there seem to be several types of inconsistency. The present paper aims at the development of a novel metatheoretical framework which provides tools for the representation and evaluation of inconsistencies in linguistic theories. The metatheoretical model relies on a system of paraconsistent logic and distinguishes between strong and weak inconsistency. Strong inconsistency is destructive in that it leads to logical chaos. In contrast, weak inconsistency may be constructive, because it is capable of accounting for the simultaneous presence of seemingly incompatible structures. However, paraconsistent logic cannot grasp the dynamism of the emergence and resolution of weak inconsistencies. Therefore, the metatheoretical approach is extended to plausible argumentation. The workability of this metatheoretical model is tested with the help of a detailed case study on an analysis of discontinuous constituents in Government-Binding Theory.
\end{abstract}

Keywords: generative syntax, inconsistency, paraconsistency, plausible argumentation

\section{Introduction}

Since the advent of Government-Binding Theory, Chomsky has continuously emphasised the necessity of dealing with inconsistencies in generative linguistics. For example, in $O n$ Binding he writes that

"[a]pparent counterexamples and unexplained phenomena should be carefully noted, but it is often rational to put them aside pending further study when principles of a certain degree of explanatory power are at stake. How to make such judgements is not at all obvious: there are no clear criteria for doing so. [...] But this contingency of rational inquiry should be no more disturbing in the study of language than it is in the natural sciences." (Chomsky 1980: 2)

During the past three decades none of the claims made here have been thought over in the generative literature as systematically as they should have been. We still do not know despite the long period of Government-Binding Theory and the rise of minimalism which also presupposes the same idea of inconsistency tolerance (see e.g. Chomsky 2002: 95) which criteria decide whether a certain inconsistency may or must not be tolerated. The present paper focuses on this issue and sets out to suggest a novel approach to the problem of inconsistency in linguistics.

To begin with, let us remind the reader of the role which the standard view of the analytical philosophy of science attributed to the law of non-contradiction in scientific theories:

"Internal inconsistency of a theory was anathema to the positivists - the mark of irrationality, the complete breakdown of logic and reason. For in standard logic and epistemology $[\ldots]$ a single inconsistency is logically and epistemically disastrous: an inconsistency anywhere instantly propagates to generate inconsistency everywhere. 
[...] Accordingly, for the positivists, and for most traditional logicians and philosophers, consistency was not one constraint on rational inquiry among others, to be balanced in cost-benefit tradeoffs with those others. Rather, it was an absolute, sine qua non for rational inquiry." (Nickles 2002: 9; emphasis as in the original)

This view has prevailed for decades among linguists, too. Thus, theoretical linguistics seems to be characterised by the presence of incompatible principles in relation to the treatment of inconsistencies.

However, the topic of inconsistency has attracted increased attention over the last few years in the philosophy of science. There have been serious attempts at its re-evaluation:

"Today, it is generally recognised that almost all scientific theories at some point in their development were either inconsistent or incompatible with other accepted findings (empirical or theoretical). A growing number of scholars moreover recognises that inconsistencies need not be disastrous for good reasoning." (Meheus 2002: VII; emphasis added)

How can the consequences of this general tendency in the philosophy of science be related to Chomsky's claims quoted above? Obviously, what is needed is the elaboration of metatheoretical criteria for the detection and evaluation of inconsistencies which, nonetheless, are not alien to and, therefore, applicable to the practice of linguistic research. However, the comprehensive discussion of such a methodology at a general level would be beyond the scope of the present paper. Therefore, we will restrict our considerations to an instructive and illuminating example by relativising them to a particular case study.

Moravcsik $(2006,2010)$ outlined a systematic typology of inconsistencies and their resolution in syntax by analysing a great number of examples. Her main hypothesis is that the differences between syntactic theories result basically from different strategies of inconsistency-resolution. Another approach was put forward in, among others, Kertész (2004), and Kertész \& Rákosi (2006, 2012). In the present paper we want to relate the two approaches in the following way: the starting point for our case study will be one of Moravcsik's (2006: $47 \mathrm{ff}$.) central examples, namely, Baltin's (1987) account of discontinuous constituents, and we will present a detailed metascientific analysis of this example by making use of the framework developed in Kertész \& Rákosi (2012).

Baltin's paper applies a particular version of Government-Binding Theory as conducted in the late 1980s. In our study, it serves merely as an object-scientific example which is expected to illustrate our metascientific approach to the structure and function of inconsistency in syntax; we do not dispute his findings and it is not our aim to criticise his analyses.

Thus the present paper will focus on the following problem:

(a) How are inconsistencies treated in Baltin's account of discontinuous constituents?

(b) Which metatheoretical framework is capable of representing these inconsistencies?

(c) How can the treatment of inconsistencies in generative syntax be evaluated?

The structure of the paper is as follows. In Section 2, we will first offer a brief survey of the structure, the function and the treatment of inconsistencies in Baltin's paper with respect to different analyses of degree word complement clauses. For lack of space, we will discuss only those aspects of his account which are relevant for the solution of $(\mathrm{P})$. In order to get an 
- at least relatively - direct and neutral picture of Baltin's approach, in this section we will not apply any specific metascientific model and will try to keep the descriptive apparatus applied to a minimum. In this way, we will obtain our answer to $(P)(a)$. Section 3 will be devoted to showing how inconsistency in scientific theories can be reconstructed by the application of Rescher \& Brandom's paraconsistent logic. Then we will apply this paraconsistent logic to Baltin's argumentation and thus obtain a solution to $(\mathrm{P})(\mathrm{b})$. We will see, however, that this result is not satisfactory, because paraconsistent logic can be applied only to the reconstruction of single contradictions; it cannot grasp the process of the continuous emergence and resolution of inconsistencies in Baltin's argumentation. Therefore, the treatment of inconsistencies cannot be evaluated on the basis of paraconsistent logic alone. Consequently, in order to solve $(\mathrm{P})(\mathrm{c})$, in Section 4 we will briefly show that the dynamism of the emergence and solution of inconsistencies can be captured if paraconsistent logic is supplemented by the tools of plausible argumentation. Finally, Section 5 will summarise our findings.

\section{On (P)(a): The emergence and the treatment of inconsistencies in Baltin (1987)}

\subsection{The basic inconsistency and four proposals to its solution}

Baltin (1987) raises the problem of discontinuous constituents in generative grammar by investigating sentences containing degree words and complement clauses such as

(1) (a) John was so tall that he hit his head on the overhead lamp.

(b) John worked on the problem too much to give up.

The syntactic analysis of examples like (1)(a) and (b) must account for two things. First, sentential complements are selected by the degree word, i.e., they are complements of the degree word. ${ }^{1}$ One of the principles of generative grammar says that complements have to be generated within the phrase whose head is their selector. The adjective, however, is not the complement of the degree word (because it can occur in a sentence without the degree word but not vice versa). From this we obtain the following structure:

$$
[\mathrm{NP} \quad \mathrm{Infl} \quad[\mathrm{V} \quad[\mathbf{Q} \quad \text { complement clause }] \mathbf{A}]]
$$

Second, sentential complements always appear in clause-final position, i.e. we have the order:

\section{(3) NP Infl $\quad$ V $\quad$ Q $\quad$ A $\quad$ complement clause}

Thus, in representing the structure of this sentence, the linear separation of the degree word and the sentential clause as well as their syntactic relatedness should be accounted for. ${ }^{2}$ That is, one has to resolve the following inconsistency which is one particular manifestation of the general problem of discontinuous constituents:

\footnotetext{
1 That is, without the degree word, sentential clauses cannot appear in the sentence. Moreover, the finiteness of the complement clause depends on the choice of the degree word $($ so + that ...; too + to ...; enough + that/to...).

2 For evidence for this claim, cf. the examples (1)-(2) and (3)-(10), respectively, in Baltin (1987: 11f.).
} 
(I) (a) Sentential complements of too, so, etc. are located between the degree word and the adjective. ${ }^{3}$

(b) Sentential complements of degree words may appear only in clause-final position. $^{4}$

The central problem of Baltin's paper is how to handle the contradictory set (I)(a) and (I)(b). (I)(a) is based on a very strong analogy between degree words and their sentential complements on the one hand, and verbs, adjectives, nouns or prepositions and their complements on the other. ${ }^{5}$ Therefore, (I)(a) is treated as a special case of a basic principle of the theory which plays an important and indispensable role in generative grammar and has been successfully applied to the description of a wide range of linguistic phenomena. (I)(b) is an inductive generalisation accounting for a characteristic of English sentences supposed to be of a specific structure. This hypothesis is characterised by Baltin as an "obvious fact that any observationally adequate grammar of English must capture" (Baltin 1987: 11).

Baltin examines and compares four proposals which have been raised in the literature in order to resolve the contradiction between (I)(a) and (I)(b).

Bresnan's proposal to handle (I) - which we will call the Proposal Based on Extraposition, (PEP) - supposes that the operation of extraposition is at work with degree word complement clauses (cf. Baltin 1987: 12f.):

(PEP) Sentential complements are generated in the deep structure between the degree word and the adjective in the case of too, so and after the degree word in the case of enough as sisters of the degree word. Then they get moved rightward and adjoined to $S$ ' in the surface structure (i.e., they are 'extraposed').

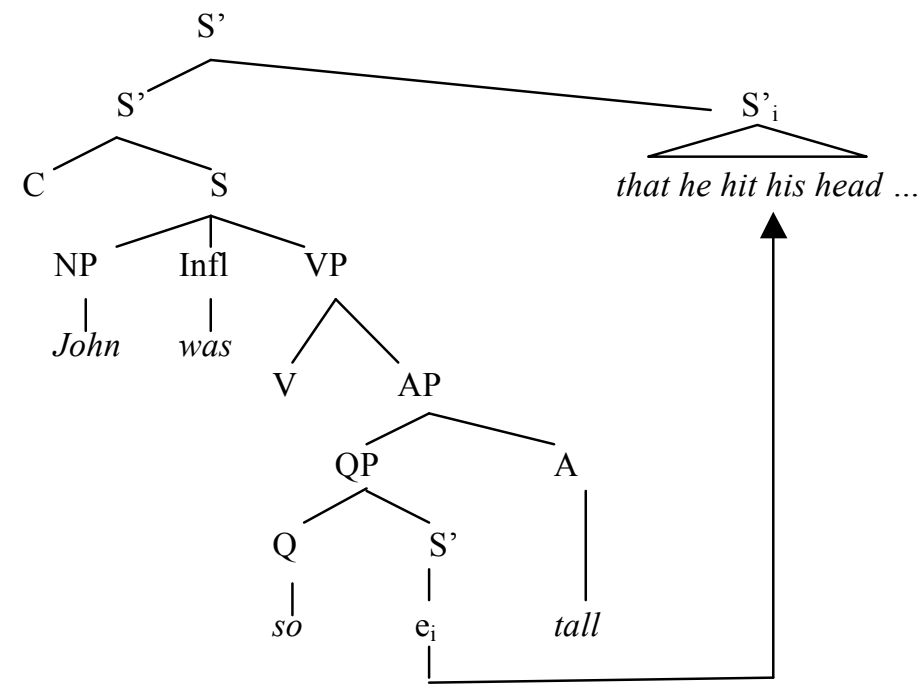

Figure 1

\footnotetext{
3 In the case of enough, the adjective precedes the degree word.

4 This formulation is vague because it does not specify in what kind of position the sentential complement appears at the end of the clause. For example, it leaves open the question of whether we have to deal with a position adjoined to $S^{\prime}$ ' from outside of $S$ or with a position within $S$, that is, within the internal structure of the clause.

5 Cf:: "[...] it seems that degree words combine with sentential complements to form linguistic units in English (and in other languages) in exactly the same fashion as do verbs, adjectives, nouns, and prepositions." (Baltin 1987: 12)
} 
The representation of the sentence in (1)(a) is as in Figure 1. (PEP) resolves the conflict between the two members of (I) by supposing that (I)(a) refers to the deep structure and (I)(b) to the surface structure.

The second proposal - which will be dubbed the Proposal Based on Discontinuous Constituency, (PDC) - is the following (cf. Baltin 1987: 15ff.):

(PDC) Degree word complements form deep- and surface structure discontinuous constituents with degree words. This relationship is always clause-bounded.

On the basis of (PDC), the surface structure of (1)(a) presents itself as in Figure 2.

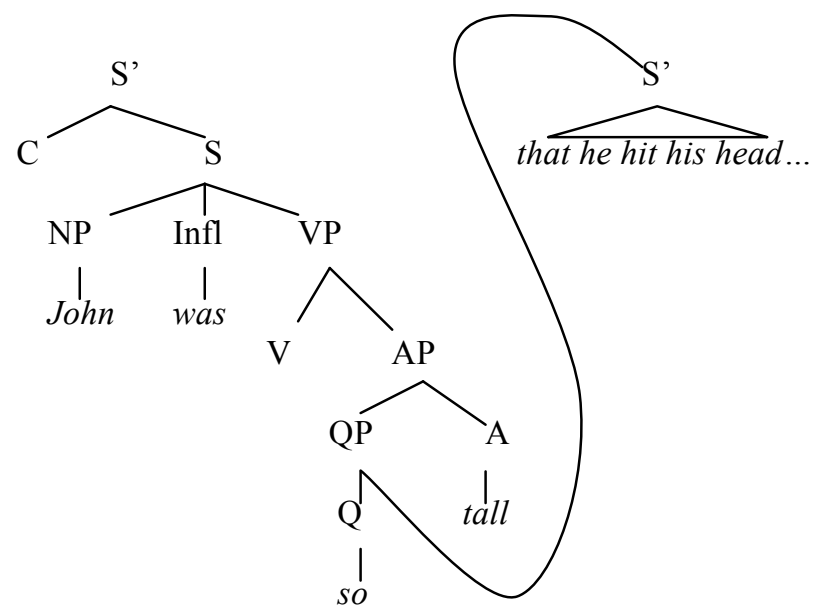

Figure 2

(PDC) resolves the conflict between (I)(a) and (b) by stating that degree word complements are thematically linked to Q but in the linear ordering of the words, they are separated. Thus, both their syntactic relatedness and their linear separation are accounted for, as witnessed by their representation in Figure 2.

The third proposal (cf. Baltin 1987: 22) will be called the Proposal Based on Multidomination, (PMD):

(PMD) Degree word complement clauses are multidominated: they constitute discontinuous constituents with the degree word (and are dominated by QP), and at the same time, they are dominated by the whole sentence.

This solution suggests representing both structures by combining them in a single tree (see Figure 3). 


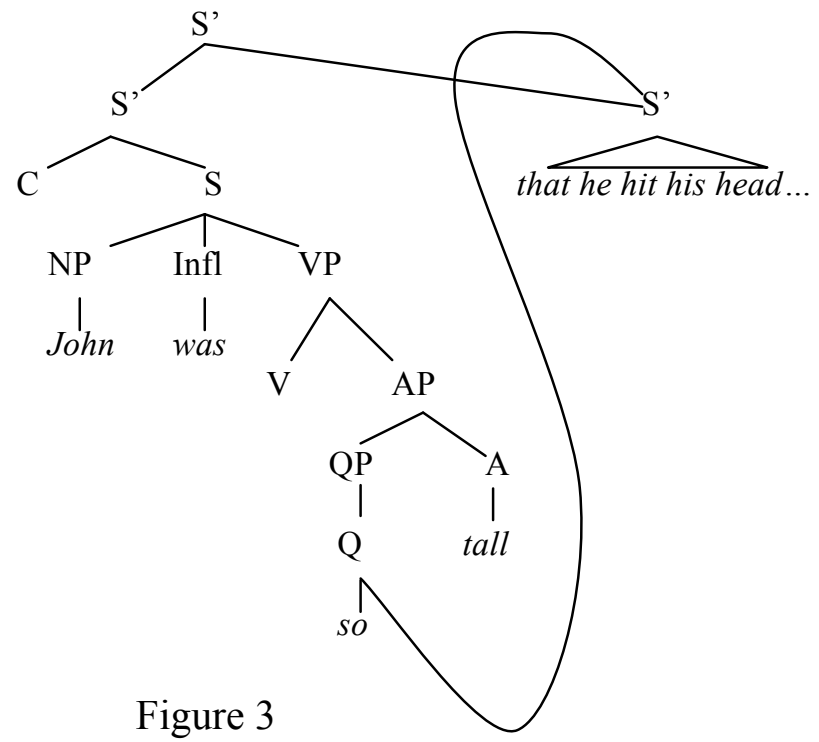

As Figure 3 shows, the complement clause is a sister of the degree word Q and, at the same time, it is also a sister of S'. Accordingly, the two analyses coexist inseparably and are maintained for the very same entity and at the very same time.

According to the fourth proposal, the conflict between (I)(a) and (b) can be resolved by the hypothesis which we call in the present paper the Proposal Based on Double Representation, (PDR):

(PDR) Sentences containing degree word complement clauses require two distinct representations: a representation for the thematic structure governing the application of transformations, where there is deep and surface discontinuous constituency between the degree word and the complement clause and a representation which is the domain for binding theory, where the degree word complement is dominated solely by the whole sentence.

(PDR) is based on double representation which keeps but also separates the structures obtained by the application of the two proposals, (PEP) and (PDC). See Figure 4 for the representation of the thematic structure governing the application of transformations and Figure 5 for the representation to which the binding theory applies (cf. Baltin 1987: 23).

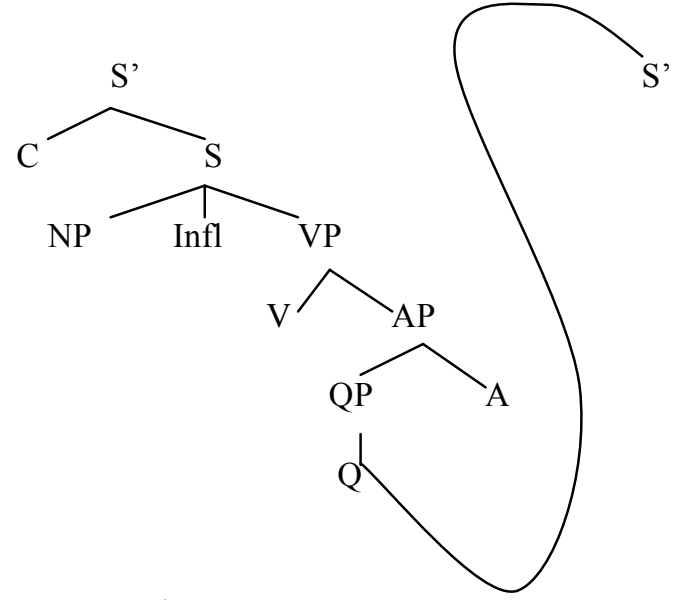

Figure 4 


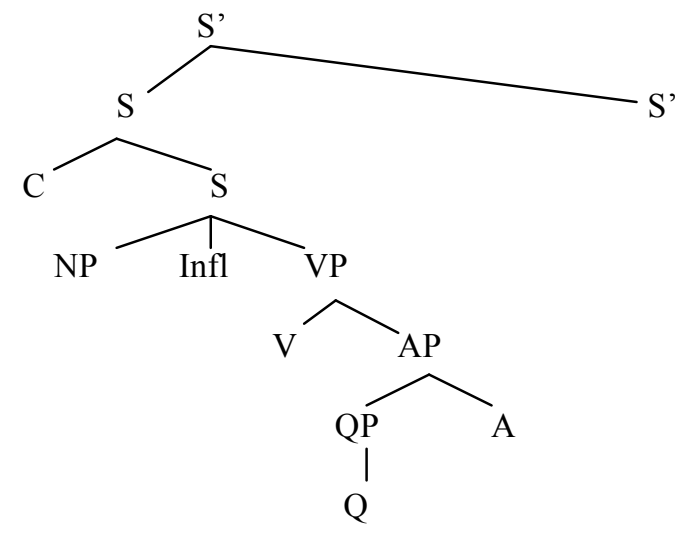

Figure 5

That is, on the one hand degree word complements constitute discontinuous constituents with the degree word (at the thematic structure), and on the other they are adjoined to the sentence from outside, that is, they are not dominated by QP but by S' (at the structure for binding relations). The contradiction (I) has been resolved here by a seemingly very simple technique: by separating the conditions under which claims referring to thematic roles and those applying to binding relations can be checked.

One of the most important characteristics of the presented proposals to handle (I) is that neither of the conflicting statements is given up but both of them are kept. This strategy seems to be fundamentally different both from Chomsky's methodological stance and from the requirement of the analytical philosophy of science cited in Section 1. This means that (PEP), (PMD), (PDC), and (PDR) do not pursue the often applied strategy related to inconsistencies, namely, the rejection of one of the conflicting statements. Moreover, neither can they be characterised in such a way that they put aside a relatively small group of counter-examples temporarily in the hope that further research will clarify their behaviour as Chomsky proposes. Instead, all four proposals consider (I) as a conflict between equally wellfounded and non-revisable hypotheses, and share the strategy of keeping both members of the inconsistency. (PEP), (PDC) and (PDR) keep both members of the contradictory pair (I) by separating them. (PMD), however, tries to maintain the two contradictory statements simultaneously, at the same level of representation and at the same time.

\subsection{The emergence of further inconsistencies}

Another striking feature of the four proposals is that they trigger further inconsistencies. Thus, Baltin's argumentation can be characterised as a process of the continuous emergence of inconsistencies and of various attempts at their resolution by examining the explanatory power and the failure of the proposals at issue. In this Subsection we will present Baltin's argumentation, but we will reduce his train of thought to the essentials by omitting several of the details and (pro and contra) sub-arguments presented in his paper.

Analyses of sentences containing both a degree word complement clause and a relative clause extraposed from subject position provide arguments for the tenability of (PEP). Since there is evidence that the relative clause will be adjoined to $S$, and by virtue of (PEP), the degree word complement clause is adjoined to $\mathrm{S}$ ', the relative clause has to precede the degree word complement clause. (4)(a) and (b) provide evidence for the correctness of this prediction (cf. Baltin 1987: 14): 
(4) (a) People were so angry who knew John that they refused to participate.

(b) *People were so angry that they refused to participate who knew John.

A second argument in favour of (PEP) stems from a principle of generative grammar, which requires that if a pronoun is co-referent with a noun, then the former cannot c-command the latter. ${ }^{6}$ Consequently, in (5) the degree word complement clause has to be attached to a higher node than the node dominating the pronoun (cf. Baltin 1987: 14):

I told her ${ }_{i}$ that so many people attended last year's concert that I made Mary nervous.

However, (PEP) leads to contradictions which we will call Inconsistencies Related to the Proposal Based on Extraposition and abbreviate as (IEP1)-(IEP4).

(IEP1) stems from the conflict between Ross's Right Roof Constraint and the hypothesis (PEP). It is as follows.

(IEP1) (a) Elements moving rightward cannot be moved out of the clause in which they originate.

(b) Extraposition moves the degree word complement clause rightward out of the given clause.

Secondly, (PEP) states that degree word complement clauses are moved by a transformation to the end of the sentence. From this it follows that every sentential complement should be associated with exactly one degree word. (6)(a), however, is a counter-example to this statement:

So many people have read so many books so often that it's hard to keep up with them.

Moreover, if there are many degree words in a sentence, then the same amount of complement clauses should appear in clause-final position. (6)(b), however, contradicts this prediction.

(b) *So many people have read so many books so often that they outnumbered us that the library was practically empty.

Therefore, (PEP) together with certain hypotheses of generative grammar makes false predictions:

(IEP2) (a) Sentential complements can be associated with exactly one degree word, and many complement clauses are allowed in the presence of many degree words.

(b) There are cases where multiple associations between a sentential complement and many degree words are possible; further, sentences are ungrammatical if many degree word complement clauses appear in clause-final position. ${ }^{7}$

\footnotetext{
6 Node $A$ c-commands node $B$ if the first branching node dominating $A$ also dominates $B$ and neither $A$ nor $B$ dominate each other.

7 That is, only one degree word complement clause is possible in a sentence, independently of the number of the degree words included by the clause.
} 
Third, from the Condition on Extraction Domains it follows that extraction is allowed from a degree word complement clause only if it is within the degree word's maximal projection, that is, if it is contained by the QP at the surface structure as well (see Baltin 1987: 17). Since according to (PEP), degree word complements are moved out of the QP and are adjoined to a higher node in the surface structure, (7) should be ungrammatical:

Who was he too angry to visit $?$

This is, however, not the case. This conflict leads to (IEP3):

(IEP3) (a) Extraction is not allowed from the sentential complement of degree words.

(b) In some cases, extraction from sentential complements of degree words is possible.

Fourth, Guéron \& May (1984) proposed to check the head-complement relation not at the surface structure but at the logical form. Although this hypothesis, together with (PEP) might explain (7), it cannot account for the ungrammaticality of (8) in Baltin's view:

\section{*Which prize did the teachers think that he $e_{i}$ was too arrogant to consider John for ?}

This contradiction can be summarised as (IEP4):

(IEP4) (a) Extraction is allowed from the sentential complement of a degree word if the degree word complement is adjoined to a higher clause than the clause containing the degree word - but at logical form, the degree word properly governs the degree word complement.

(b) In the case of sentential complements of degree words where the degree word complement is attached to a higher node than the clause containing the degree word, extraction is not possible.

Therefore, whether we accept Guéron and May's proposal or not, (PEP) is not capable of explaining the contrast between (7) and (8).

From this Baltin concludes that (PEP) is insufficient and he raises another solution to (I) which is capable of explaining the examples (6)-(8), namely, (PDC).

However, Baltin (1987: 20) remarks that (PDC) is not satisfactory, either. It leads to inconsistencies as well, which will be dubbed Inconsistencies Related to the Proposal Based on Discontinuous Constituency. First, in sentences containing both a degree word complement clause and a relative clause extraposed from subject position like (4), (PDC) does not stipulate the order of these clauses:

(IDC1)(a) In sentences containing both a degree word complement clause and a relative clause extraposed from subject position, the order of these two clauses is unconstrained.

(b) In sentences containing both a degree word complement clause and a relative clause extraposed from subject position, the latter has to precede the former.

Second, Chomsky's binding theory requires anaphors to be c-commanded by their antecedents. Thus, if (PDC) were true, anaphors within a degree complement should be able to be bound by the matrix subject. But (9) refutes this prediction (cf. Baltin 1987: 20f.): 
(a) *They were too partisan for each other to be convinced.

(b) They were too partisan to convince each other.

Namely, if the degree word complement clause were dominated by QP, then the subject of the main clause in (9)(a) should bind the reciprocal. In (9)(b), the reciprocal is bound by PRO, which, however, does not require to be c-commanded by the subject of the main clause. We obtain in both cases that the degree word complement clause cannot be within the VP. These findings yield (IDC2):

(IDC2)(a) Anaphors within degree complement clauses can be bound by the matrix subject.

(b) Anaphors within degree complement clauses cannot be bound by the matrix subject.

Baltin (1987: 22) argues that degree word complements behave paradoxically: extraction from them can be explained only by supposing discontinuous constituency, while binding relations require that they are attached to a higher position. Therefore, first he raises the hypothesis that these two structures should be simply combined with each other within a single representation and raises (PMD).

As Baltin remarks, however, (PMD) leads to faulty predictions because (PDC) inherits not only the explanatory power of the two models which it combines, but preserves their shortcomings as well. Therefore, the inconsistency captured by (IDC2), for example, emerges in this case again and will be referred to as Inconsistency Related to the Proposal Based on Multidomination:

(IMD1) (a) Anaphors within degree complement clauses can be bound by the matrix subject.

(b) Anaphors within degree complement clauses cannot be bound by the matrix subject.

(PMD) also infringes a basic principle of generative grammar that prohibits multidomination:

(IMD2) (a) Every node can be immediately dominated by only one node; that is, every node has only one mother node.

(b) Degree word complements are immediately dominated by two nodes; that is, they have two mother nodes.

Therefore, Baltin rejects (PMD) immediately. As he remarks, however, the problem is that one can give up neither member of (PMD) because both of them are needed to explain examples like (4)-(9). Nevertheless, he also realises that they behave complementarily in the sense that each of them can explain linguistic data which lead to a failure in the case of the other proposal. Thus, he raises (PDR).

However, Baltin (1987: 24f.) himself realises that (PDR) is problematic in two respects. First, the grammar containing (PDR) does not involve hypotheses which would restrict discontinuous constituency to degree word complements. Therefore, a hypothesis system containing (PDR) overgeneralises, because there are cases in which complements cannot be separated from their selector, as the Inconsistency Related to the Proposal Based on Double Representation captures: 
(IDR) (a) Discontinuous constituency allows the head, its complements and other constituents within this phrase to appear in any arbitrary order.

(b) Structures like a fond man of Sally are not grammatical.

Second, Baltin cannot give a satisfactory answer to the question of what the origin of the distinction between extraposed and non-extraposed positions in relation to degree word complements is.

Table 1 summarises the proposals to solve (I) and the inconsistencies related to them:

\begin{tabular}{|l|l|}
\hline Proposal Based on Extraposition & (PEP) \\
\hline - conflict with Ross's Right Roof Constraint & (IEP1) \\
\hline $\begin{array}{l}\text { conflict with the hypothesis of 1:1-relation between sentential complements and degree } \\
\text { words }\end{array}$ & (IEP2) \\
\hline - conflict with the Condition on Extraction Domains & (IEP3) \\
\hline - conflict with the hypothesis of checking the head-complement relation at the logical form & (IEP4) \\
\hline Proposal Based on Discontinuous Constituency & (PDC) \\
\hline $\begin{array}{l}\text { conflict with the hypothesis of the free ordering of degree word complements and relative } \\
\text { clauses extraposed }\end{array}$ & (IDC1) \\
\hline - conflict with Chomsky's Binding Theory & (IDC2) \\
\hline Proposal Based on Multidomination & (PMD) \\
\hline - conflict with Chomsky's Binding Theory & (IMD1) \\
\hline - conflict with the prohibition of multidomination & (IMD2) \\
\hline Proposal Based on Double Representation & (PDR) \\
\hline - conflict with linguistic data & (IDR) \\
\hline
\end{tabular}

\section{Table 1}

On the basis of the above considerations, three further very important points with respect to the handling of inconsistencies in Baltin's paper can be highlighted.

First, they have a heuristic function insofar as they motivate the elaboration of new hypotheses and theory-versions:

- (IEP1)-(IEP4) indicate that the hypothesis of moving out the degree word complement from the sentence and adjoining it to a higher node is incorrect. This led to the rejection of (PEP) and to the introduction of (PDC).

- (IEP3) impelled the partial revision of the theory, because Guéron and May tried to replace some parts of generative grammar by new hypotheses.

- (IDC1)-(IDC2) have shown not only that the acceptance of (PDC) leads to problems but also revealed that there seems to be a complementary relationship between (PEP) and (PDC): they can explain phenomena which are problematic for the rival proposal and fail where the other proposal succeeds.

- This finding motivated the suggestion that (PEP) and (PDC) should be treated not as rival hypotheses but as hypotheses which have to be maintained simultaneously. Thus, Baltin raised (PMD) which combines both proposals; but the circumstance that (PMD) is not capable of avoiding the inconsistencies which emerged with respect to (PDC) resulted in its prompt rejection.

- As a consequence, Baltin came up with the idea of keeping the structures resulting from the application of the two incompatible hypotheses (PEP) and (PDC), respectively, in such a way that their field of application is strictly separated. This led to the elaboration of (PDR). 
Second, Baltin rejects (PEP), (PDC) and (PMD) not because they keep the contradictory claims (I)(a) and (I)(b) but he deems them unsatisfactory since they lead to further inconsistencies and other problems. Nevertheless, he does not give up (PEP) and (PDC) immediately after having found out that they lead to further inconsistencies, but only in possession of a better rival proposal. This is, of course, at variance with the requirement of the standard view of the analytical philosophy of science according to which hypotheses leading to contradictions should be given up promptly. Chomsky's proposal is not capable of providing a thorough explanation of the treatment of these inconsistencies, either. Baltin does not simply put them aside in the hope of some future explanation and he does not go on with the given hypothesis (PEP)/(PDC)/(PMD)/(PDR), but he evaluates and weighs up these inconsistencies together as parts of rival systems of hypotheses around (PEP), (PDC), (PMD) and (PDR). From this we can conclude that inconsistencies fulfil their role in Baltin's argumentation not in isolation but in a context-sensitive way, in relation to the alternative hypotheses at hand and to the other inconsistencies.

Third, Baltin does not propose a new hypothesis after rejecting the first two hypotheses but he maintains the structures obtained by the application of (PEP) and (PDC), and builds them into (PDR). Thus, the solution he prefers, that is, (PDR), makes use of the strategy of the maintenance of two conflicting hypotheses by separating their field of application twice: first, with respect to (I)(a) and (b), and second, at a higher level, in connection with possible solutions to (I), that is, with respect to (PEP) and (PDC).

\subsection{Summary}

From the above considerations we can conclude that the picture emerging from the reconstruction of Baltin's argumentation undeniably clashes with the norms of the standard view of the analytical philosophy of science at several points, and cannot be explained with the help of Chomsky's methodological stance, either.

Baltin treats the inconsistencies (IE1)-(IDR) not as fatal failures requiring a prompt treatment. Rather, he considers them as counter-arguments which motivate the search for novel hypotheses or open problems which will hopefully be solved. Accordingly, these inconsistencies play a dual function in the development of his approach. On the one hand, the counter-examples question (but do not disprove conclusively) the acceptability of the given hypothesis; that is, they provide counter-arguments against the given hypothesis. On the other hand, they stimulate the search for new proposals.

As a consequence, the role of the "confirming" and the "refuting" examples is not strictly asymmetrical. In the case of (PEP), (PDC) etc., Baltin enumerates many arguments for and against them. Although one falsifying case should suffice to make the given hypothesis unacceptable, Baltin pursues another strategy. He investigates both sides, that is, he considers arguments supporting a given hypothesis, too, and he tries to reveal the strengths and weaknesses of this hypothesis and compare them with those of its rivals.

A further important characteristic of Baltin's argumentation is that although the theoryversions based on (PEP), (PDC) and (PDR) have a similar structure in so far as they keep (and separate) both (I)(a) and (I)(b) and contain further contradictions as well, they are evaluated differently: Baltin prefers (PDR) against (PEP) or (PDC). Neither the (systematically separated) maintenance of contradictory hypotheses (cf. Subsection 2.1) nor differences in the evaluation of inconsistent systems of hypotheses can be grasped in the standard view.

Our next task is to find a metatheoretical approach that is capable of accounting for these aspects of inconsistency. 


\section{On (P)(b): Paraconsistency 3.1. Introductory remarks}

The standard view of the analytical philosophy of science reconstructs theories with the help of classical logic. However, classical logic cannot explain how it is possible that there are scientific theories which are contradictory but functional, non-trivial and non-chaotic, because classical logic maintains the principle of ex contradictio quodlibet, i.e. anything follows from contradictory premises. This means that for every proposition $A$ and $B,\{A, \sim A\}$ $\Rightarrow B$. Thus, for classical logic all inconsistencies are equally disastrous and intolerable.

In the second half of the twentieth century several attempts were made to develop nonclassical logics in which it is not the case that anything follows from a pair of contradictory statements. More precisely, let $\vDash$ be the relation of logical consequence, defined either semantically (that is, in terms of truth conditions) and symbolised by $\Rightarrow$, or syntactically (that is, in terms of derivability), symbolised by $\vdash . \vDash$ is explosive if and only if for every statement $A$ and $B,\{A, \sim A\} \vDash B$. Classical logic is explosive. A logic is said to be paraconsistent if and only if its relation of logical consequence is not explosive. (For recent surveys see, for example, Priest 2002a,b, Meheus (ed.) 2002, Priest et al. (eds.) 2004, Priest \& Tanaka 2009, Tanaka et al. (eds.) 2013 etc.). The relevance of paraconsistent logics is, among others, that they facilitate the consistent reconstruction of an inconsistent system of statements. These systems are well suited to explain the circumstance that in many cases inconsistent theories are not trivial. Some version of paraconsistent logic seems to be inevitable whenever one deals with inconsistency. Thus, for example, automated reasoning (information processing) or belief revision needs to be supplemented by a paraconsistent system (Priest \& Tanaka 2009: Sections 2.3-2.4, Priest 2001).

It is important to distinguish paraconsistency from dialetheism. While paraconsistency merely maintains that inconsistency does not necessarily lead to triviality i.e. to the possibility of inferring any arbitrary statement from a contradictory pair of statements, dialetheism claims that there are true contradictions. ${ }^{8}$ We will suggest the application of a paraconsistent logic to $(\mathrm{P})(\mathrm{b})$, which does not amount to dialetheism, i.e. does not claim that the contradictions that obtain in the system of statements are true.

With the logical tools thus introduced we intend to meet the following new desideratum of the philosophy of science:

"[...] we are left with the task of better understanding how inconsistency and neighboring kinds of incompatibility are tamed in scientific practice and the corresponding task of better modeling idealised practice in the form of inconsistencytolerant logics and methodologies." (Nickles 2002: 2)

In the next subsection, we will show how this can be done.

\footnotetext{
8 " [...] the view that a consequence relation should be paraconsistent does not entail the view that there are true contradictions. Paraconsistency is a property of an inference relation whereas dialetheism is a view about some sentences (or propositions, statements, utterances or whatever, that can be thought of as truth-bearers). The fact that one can define a non-explosive consequence relation does not mean that some sentences are true. That is, the fact that one can construct a model where a contradiction holds but not every sentence of the language holds (or, if the model theory is given intensionally, where this is the case at some world) does not mean that the contradiction is true per se. Hence paraconsistency must be distinguished from dialetheism." (Priest \& Tanaka 2009)
} 


\subsection{Rescher and Brandom's paraconsistent logic}

One of the most fruitful attempts at the elaboration of a paraconsistent logic is Rescher and Brandom's seminal book The Logic of Inconsistency (Rescher \& Brandom 1980). ${ }^{9}$

The authors presuppose a Kripke-semantics, and they introduce, among other things, the operation of superposition on the set of possible worlds which relates possible worlds disjunctively: ${ }^{10}$

(10) Superposition: Let $w_{1}$ and $w_{2}$ be two possible worlds. $w_{1} \cup w_{2}$ is that world such that, for any statement $A, A$ obtains in this world if and only if $A$ obtains either in $w_{1}$ or in $w_{2}:^{11}$

$$
[A]_{w_{1} \odot_{w_{2}}}=+\operatorname{iff}[A]_{w_{1}}=+ \text { or }[A]_{w_{2}}=+
$$

See Table 2.

\footnotetext{
9 The choice of Rescher and Brandom's model has been motivated by several considerations. First, our pmodel of linguistic theorising - which we will introduce in Section 4 - is partially rooted in Rescher's approach to plausible argumentation. Therefore, the application of Rescher and Brandom's paraconsistent logic is quite natural. Second, this logic is especially useful because it also challenges the principle of the excluded middle (which we do not discuss in this paper but which is important for other applications of the p-model). Thus it provides us with a unified treatment of deviances from two basic principles of classical logic. Third, there are several systems of paraconsistent logic, which are built on different background assumptions depending on the particular considerations motivating them. For example, according to Priest \& Tanaka (2009), Tanaka et al. (2013), discussive logic (Jaśkowski 1948) intends to model discourses with differences of opinion; preservatorism (Scotch \& Jennings 1980) aims at modelling information flow between mutually inconsistent but inherently consistent fragments of a theory; the basic idea of adaptive logics (Batens 2001, 2007) is that inconsistency is a relatively rare phenomenon, thus rules of inference have to be extended with a few supplementary strategies which make it possible to apply the inference rules to the "abnormalities"; logics of formal inconsistency (da Costa 1974, Carnielli et al. 2007) are a valuable endeavour to avoid isolation of inconsistency from the consistent parts of the given theory with the help of introducing metatheoretical notions of (in)consistency in the object language. Thus, it may be-a reasonable option to examine in what way and to what extent different paraconsistent logics are compatible with the p-model. Nevertheless, the p-model is not compatible with every paraconsistent system. For example, Carnielli et al.'s (1991) semantics of translations, which aims at modelling reasoning by default, is based on assumptions that are at certain points at variance with the p-model's interpretation of plausibility values. While Carnielli et al. stipulate that statements may be deemed definitely false on the basis of some piece of evidence but "positive evidence of trueness is not possible" (Carnielli et al. 1991: 534), the p-model enables both extremes and a continuum of intermediate values ranging from neutral plausibility to truth with certainty as well as from falsity with certainty to neutral plausibility (see Section 4.1 and Kertész \& Rákosi 2012: 63ff.). Thus, if one decides for a paraconsistent logic which is incompatible with the p-model at the outset, then another metatheoretical model of linguistic theorising must be chosen.

See also Kertész (2004) and (2012) for further applications of Rescher and Brandom's paraconsistent logic to linguistics.

10 Another operation Rescher and Brandom introduce is schematisation which is intended to capture situations in which the law of the excluded middle is not valid. With respect to the treatment of inconsistency, it is only superposition that is relevant; therefore, we will not deal with schematisation.

$11[A]_{w}=+$ and $[A]_{w}=-$ are contradictory: $A$ either obtains in a world or not.
} 


\begin{tabular}{|c|c|c|}
\hline$[A]_{w_{1}}$ & {$[A]_{w_{2}}$} & {$[A]_{w_{1}} \cup_{w_{2}}$} \\
\hline+ & + & + \\
\hline+ & - & + \\
\hline- & + & + \\
\hline- & - & - \\
\hline
\end{tabular}

Table 2

We will call $w=w_{1} \cup w_{2}$ the superposed possible world, and $w_{1}$ and $w_{2}$ the component possible worlds. Possible worlds in which the principles of classical logic hold are called standard possible worlds. Rescher \& Brandom (1980: 15) define the truth operator as

(11) $A$ is true in $w$ if and only if $A$ obtains in $w$, i.e. $\mathrm{t}_{\mathrm{w}}(A)$ iff $[A]_{w}=+$.

From (10) and (11) we get that with superposed worlds, $A$ is true in $w_{1} \uplus w_{2}$ if and only if it is true at least in one of the component worlds. Four properties of Rescher and Brandom's paraconsistent logic are relevant for the solution of our problem $(\mathrm{P})(\mathrm{b})$. First, superposed worlds are non-standard, ${ }^{12}$ because it may be the case that both $A$ and its negation are true in such a world. For example, let us suppose that $w_{1}$ and $w_{2}$ are standard worlds, and $[A]_{w_{1}}=+$, $[A]_{w_{2}}=-$. Since in $w_{1}$ and $w_{2}$ the principles of standard logic prevail, $[\sim A]_{w_{1}}=-$ and $[\sim A]_{w_{2}}=$ + . From $[A]_{w_{1}}=+$ we obtain that $t_{w_{1}}(A)$ and from $[\sim A]_{w_{2}}=+$ it follows that $t_{w_{2}}(\sim A)$. According to (10) and (11), the former leads to $t_{w_{1} \cup_{w_{2}}}(A)$ and the latter to $t_{w_{1} \omega_{w_{2}}}(\sim A)$; that is, both $A$ and $\sim A$ are true in the superposed world $w .^{13}$

Second, from the fact that two statements are true in a superposed world, it does not follow that their conjunction is true in this world as well. It is easy to show that this is so: if $A$ is true in the component world $w_{1}$ but false in $w_{2}$ and $B$ is true in $w_{2}$ but false in $w_{1}$, then both $A$ and $B$ will be true in $w_{1} \cup w_{2}$ but $A \& B$ will not, since the latter obtains in none of the component worlds.

Third, in these worlds the semantic consequence relation ' $\Rightarrow$ ' is not explosive. Accordingly, it is possible to have both $A$ and $\sim A$ in a superposed world $w$, but from $t_{w}(A)$ and $t_{w}(\sim A)$ we cannot conclude that $t_{w}(B)$.

Fourth, the classical and the paraconsistent treatment of inconsistency are different. In standard worlds, any arbitrary statement $B$ can be inferred from a contradiction. In superposed worlds, however, there is a fundamental difference between two kinds of inconsistency:

(12) Weak inconsistency: "To accept the prospect that for some genuinely possible world $w: t_{\mathrm{w}}(A)$ and $t_{\mathrm{w}}(\sim A)$, for some $A . "$ (Rescher \& Brandom 1980: 24)

If $w=w_{1} \cup w_{2}$ and both component worlds are standard worlds, then (12) means that $A$ obtains in $w_{1}$ but it does not obtain in $w_{2}$, and $\sim A$ obtains in $w_{2}$ but it does not obtain in $w_{1}$ or conversely. It is not the case that ex contradictio quodlibet.

(13) Strong inconsistency: "To accept the prospect that for some genuinely possible world $w: t_{\mathrm{w}}(A \& \sim A)$, for some $A . "$ (Rescher \& Brandom 1980: 24)

\footnotetext{
12 In Rescher and Brandom's system there is another kind of non-standard possible world, too, in which the law of excluded middle is not valid.

13 Rescher and Brandom introduce ' $A$ is false' as ' $\sim A$ is true'. Therefore, in superposed worlds it may happen that a statement is both true and false (and in schematic worlds that it is neither true nor false).
} 
If $w=w_{1} \cup w_{2}$, then (13) means according to (10) and (11) that $A \& \sim A$ obtains in $w_{1}$ or this conjunction obtains in $w_{2}$. Thus, in a strongly inconsistent word it is the case that ex contradictio quodlibet.

In contrast, classical logic cannot differentiate between (12) and (13). Therefore in standard worlds, these two versions of inconsistency are equivalent and lead inevitably to logical chaos. In superposed worlds, however, (12) is harmless because the contradictory statements cannot serve as premises of conclusions. As opposed to this, (13) is harmful because nothing prevents one drawing conclusions from contradictory premises within the same possible world.

In sum, Rescher and Brandom's approach suggests the following general treatment of inconsistencies:

(14) (a) The separate maintenance of the two contradictory claims leads to weak inconsistency. If the two contradictory claims hold under different circumstances, then on the meta-level they can be represented in two different possible worlds. The superposition of the latter corresponds to the whole theory which does not contain the conjunction of the two claims.

(b) The simultaneous maintenance of the two contradictory claims results in strong inconsistency. This means that if the two claims contradicting each other hold under the same conditions, then on the meta-level they have to be represented within the same possible world which contains the conjunction of the two claims.

(c) Only weak inconsistency can be tolerated and strong inconsistency has to be rejected.

At this point the question is whether the application of (14) to Baltin's analysis yields the solution to the problem $(\mathrm{P})(\mathrm{b})$.

\subsection{The paraconsistent reconstruction of Baltin's approach 3.3.1. The reconstruction of (PEP), (PDC), (PDR) and (PMD)}

The metascientific reconstruction of the different strategies to treat (I) seems to be very simple along the lines of (14). ${ }^{14}$ In the case of (PEP), we obtain (15):

(15) (a) Let the following statements be given:

$A=$ The degree word, the sentential complement and the adjective are cogenerated as a continuous constituent. (Cf. Baltin 1987: 12)

$B=\quad$ Sentential complements are adjoined to the sentence.

(b) Let the possible world $w_{1}$ be the deep structure and the possible world $w_{2}$ the surface structure. ${ }^{15}$ If $w$ stands for the whole theory, then $w=w_{1} \odot w_{2}$.

(c) $A$ obtains in $w_{1}$ but does not obtain in $w_{2}$ and $B$ obtains in $w_{2}$ but does not obtain in $w_{1}$. Consequently, in $w$ both $A$ and $B$ are true, but $A \& B$ is true neither in $w_{1}$ nor in $w_{2}$.

\footnotetext{
14 In connection with these reconstructions, it is important to remind the reader that ' $A$ is true in $w$ ' means in Rescher and Brandom's paraconsistent logic that $A$ obtains in the possible world $w$; cf. (11). ' $A$ is false in $w$ ' means ' $\sim A$ is true in $w$ ', that is, $\sim A$ obtains in this possible world.

${ }_{15}$ Or to be more precise: Let the possible world $w_{1}$ be the set of the statements related to the deep structure and $w_{2}$ be the set of the statements related to the surface structure.
} 
(d) In the possible world $w_{1},(\mathrm{I})(\mathrm{a})$ is true, i.e., sentential complements are located between the degree word and the adjective, because (I)(a) can be inferred from $A$. Similarly, in the possible world $w_{2},(\mathrm{I})(\mathrm{b})$ is true, i.e., sentential complements appear in clause-final position, because (I)(b) can be inferred from $B$. However, (I)(a) does not obtain in $w_{2}$ and (I)(b) does not obtain in $w_{1}$.

(e) From this we can conclude that while both (I)(a) and (I)(b) are true in $w$, their conjunction, i.e. (I)(a) \& (I)(b) is not.

(f) This means that (I)(a) and (I)(b) are only weakly inconsistent in the sense of (12).

(PDC) can be reconstructed similarly, only (15)(a) and (b) have to be replaced by the following hypotheses:

(16) (a) $A=$ Sentential complements are dominated by QP and included in it.

$B=$ Sentential complements are dominated by QP and can be found in a clause-final position without being adjoined to $S^{\prime}$.

(b) Let the possible world $w_{1}$ be the thematic relations and the possible world $w_{2}$ the linear ordering of the words.

With (PDR), the situation is more complex, since 3 possible worlds are needed for the reconstruction of this strategy:

(17) (a) Let the following statements be given:

$A=$ Sentential complements are dominated by QP and included in it.

$B=\quad$ Sentential complements are dominated by QP and can be found in a clause-final position without being adjoined to $S$ '.

$C=$ Sentential complements are not dominated by QP and are adjoined to S'.

(b) Let the possible world $w_{1}$ be the set of the statements concerning the thematic structure of sentences, the possible world $w_{2}$ be the statements describing the linear ordering of words within the $S$ containing the degree word, and $w_{3}$ be the set of the statements which refer to the binding relations. If $w$ stands for the whole theory, then $w=w_{1} \odot w_{2} \odot w_{3}{ }^{16}$

(c) $A$ obtains in $w_{1}$ but does not obtain in $w_{2}$ and $w_{3} ; B$ obtains in $w_{2}$ but does not obtain in $w_{1}$ and $w_{3}$; $C$ obtains in $w_{3}$ but does not obtain in $w_{1}$ and $w_{2}$. Consequently, $A, B$ and $C$ are true in $w$, but $A \& B \& C$ or any conjunction of two of these statements is true neither in $w_{1}$ nor in $w_{2}$ nor in $w_{3}$.

(d) In the possible world $w_{1}$, (I)(a) is true, i.e., sentential complements are located between the degree word and the adjective, because this statement can be inferred from $A$. Similarly, in the possible worlds $w_{2}$ and $w_{3}$, (I)(b) is true, i.e., sentential complements appear in clause-final position, because it can be inferred both from $B$ and $C$. However, (I)(a) does not obtain in $w_{2}$ and $w_{3}$, and (I)(b) does not obtain in $w_{1}$.

(e) From this we can conclude that while both (I)(a) and (I)(b) are true in $w$, their conjunction, i.e. (I)(a) \& (I)(b) is not.

(f) This means that (I)(a) and (I)(b) are only weakly inconsistent in the sense of (12).

This reconstruction yields a possible explanation for the double-sidedness of the proposals

16 This can, of course, also be represented as $\left(w_{1} \odot w_{2}\right) \smile w_{3}$ which is more intelligible in the light of (PDR). 
(PEP), (PDC) and (PDR). On the one hand, they are workable, because they do not allow conclusions to be drawn from inconsistent premises. On the other hand, our reconstruction has shown that the two statements contradicting each other can be maintained as long as they are separated from each other; that is, they may be true only in two different possible worlds.

However, (PMD) does not allow this kind of reconstruction because the inconsistent premises do not become separated but appear in the same possible world. (PMD) can be reconstructed in Rescher and Brandom's paraconsistent logic as follows:

(a) $=(17)(a)$

(b) Let the possible world $w$ be the whole theory.

(c) $\quad A, B$ and $C$ obtain in $w$.

(d) In the possible world $w,(\mathrm{I})(\mathrm{a})$ is true, because it can be inferred from $A$. Similarly, in the possible world $w,(\mathrm{I})(\mathrm{b})$ is true, because it can be inferred from $B$ as well as from $C$.

(e) From this we can conclude that since (I)(a) and (I)(b) are simultaneously present in $w$, so their conjunction, i.e. (I)(a) \& (I)(b) obtains in $w$ as well.

(f) Accordingly, (I)(a) and (I)(b) are strongly inconsistent in the sense of (13).

At this point, we can conclude that the paraconsistent reconstruction provides a possible explanation of the strategy mentioned in Section 2.1 and at the end of Section 2.2. There we maintained that, instead of giving up at least one member of a contradictory pair of statements, in certain cases it may be entirely legitimate to keep both claims, each of which is equally well-supported. The paraconsistent reconstruction interpreted the strategy of retaining both members of an inconsistency by separating and representing them as weak inconsistency; in contrast, the strategy of retaining both members of a contradictory pair of statements without separating them was interpreted as strong inconsistency. Therefore, the fundamental difference between these two strategies could be accounted for: while the separation (weak inconsistency) does not lead to logical chaos and may be a legitimate strategy despite tolerating inconsistency, strong inconsistency is disastrous and has to be rejected automatically.

\subsubsection{The reconstruction of (IEP1), ..., (IDR)}

According to Section 2.3, Baltin regards the inconsistencies (IEP1), .., (IDR) as counterarguments which are evaluated together with other arguments and motivate the search for novel hypotheses. Thus, their paraconsistent reconstruction should mirror this finding. This is, however, not the case; the paraconsistent logic does not provide us with tools which would allow us to model this strategy. First of all, it is not clear whether these inconsistencies should be reconstructed as strong or weak ones. On the one hand, they can be easily reconstructed as strong inconsistencies. For example, in the case of (IEP2) one possibility is (19):

(19) (a) Let the following statements be given:

$A=(\mathrm{PEP})$

$B=\quad$ If the phrase XP is moved out of the phrase QP by a transformation, then XP can be related to exactly one Q.

$C=$ The sentence in (6)(a) is grammatical while the sentence in (6)(b) is not.

(b) Let the possible world $w$ be the whole theory.

(c) $\quad A, B$ and $C$ obtain in $w$. 
(d) In the possible world $w$ (IEP2)(a) is true, because it is a consequence of $A \& B$. Similarly, in the possible world $w$ (IEP2)(b) is true, because it is a consequence of $C$.

(e) From this we can conclude that since (IEP2)(a) and (IEP2)(b) are simultaneously present in $w$, the set $\{($ IEP2)(a), (IEP2)(b) $\}$ is strongly inconsistent in the sense of (13).

This proposal is, however, counterintuitive insofar as (IEP1)-(IDR) do not seem to lead to such fatal consequences as was the case with (PMD). The simultaneous maintenance of these contradictory pairs of statements does not trigger further inconsistencies. The reason for this situation is that in such cases it is possible to apply a strategy which allows for a narrowing down of the scope of the first member of these contradictions. This strategy relies on the idea of weak inconsistency. Let us consider the reconstruction of (IEP2) again:

(20) (a) $=(19)(\mathrm{a})$

(b) Let the possible world $w_{1}$ be the set of statements which describe the behaviour of sentences containing degree word complement clauses, and the possible world $w_{2}$ the set of statements which describe the behaviour of sentences which do not contain degree word complement clauses.

(c) The hypothesis in $B$ is not allowed to be applied to degree word complements. Thus, $B$ does not obtain in $w_{1}$ but it is false in this possible world. ${ }^{17}$ Therefore, in $w_{1}, A$ and $C$ are true but $B$ is false; in other words, sentences containing degree word complement clauses are treated as exceptions to $B$. In $w_{2}, B$ is true; $A$ and $C$ do not belong to this world; therefore, according to (11), they cannot be true in $w_{2}$.

(d) In the possible world $w_{1}$, (IEP2)(a) does not obtain. It is a consequence of $A$ and $B$ but $B$ is false in this world. (IEP2)(b) is true in $w_{1}$. In the possible world $w_{2}$, (IEP2)(a) and (b) are not present.

(e) From this we can conclude that (IEP2)(a) does not obtain in $w=w_{1} \cup w_{2}$ and only (IEP2)(b) is true in $w$. Therefore, the set $\{($ IEP2)(a), (IEP2)(b) $\}$ is not inconsistent in $w$.

(f) This resolution of the inconsistency between (IEP2)(a) and (IEP2)(b) is due to the finding that in $w$, the set $\{B, \sim B\}$ is weakly inconsistent, since $B$ is true in $w_{1}$ but false in $w_{2}$.

A further possible reconstruction interprets the relationship between (IEP2)(a) and (b) as weak inconsistency, too:

(21) (a) $=(19)(a)$

(b) Let the possible world $w_{1}$ be the set of the abstract (higher-level) hypotheses of generative grammar, and the possible world $w_{2}$ the set of statements based on grammaticality judgements of sentences containing degree word complements.

(c) In $w_{1}, A$ and $B$ obtain but $C$ does not because statements about the grammaticalness of sentences do not belong to this possible world. In $w_{2}, C$ obtains; $A$ and $B$ do not belong to this world; therefore, according to (11), they cannot be true in $w_{2}$.

(d) In the possible world $w_{1}$, (IEP2)(a) is true because it is a consequence of $A$ and $B$; (IEP2)(b), in contrast, does not obtain in $w_{1}$ due to the absence of $C$. In the

17 That is, $\sim B$ is present in it. 
possible world $w_{2}$, (IEP2)(a) does not obtain but (IEP2)(b) can be inferred from $C$.

(e) From this we can conclude that both (IEP2)(a) and (b) are true in $w=w_{1} \circlearrowleft$ $w_{2}$. Therefore, the set $\{(\mathrm{IEP} 2)(\mathrm{a}),(\mathrm{IEP} 2)(\mathrm{b})\}$ is weakly inconsistent.

We can reach similar results by reconstructing (IEP1)-(IDR).

\subsection{The limits of the paraconsistent reconstruction}

If we compare the results of our paraconsistent reconstruction with the characterisation of Baltin's treatment of inconsistencies as summarised in Sections 2.1-2.3, then it is easy to realise that there are aspects of Baltin's treatment of inconsistencies which cannot be explained with the help of Rescher and Brandom's paraconsistent logic.

First, although (PEP), (PDC) and (PDR) made it possible to reconstruct (I)(a) and (b) as weak inconsistency along the same lines, Baltin does not evaluate these alternatives equally: he prefers (PDR) to (PDC) and (PEP). Second, while (IEP1)-(IDC2) can also be reconstructed with the help of weak inconsistency, in these cases - in contrast to (IDR) Baltin decides in favour of giving up one of the contradictory statements. Nevertheless, he does this not immediately after the realisation of the given inconsistency in isolation but as a result of the consideration of all relevant arguments for and against the hypothesis at issue. Third, the paraconsistent reconstruction cannot grasp the heuristic function of inconsistencies, that is, their role in the motivation of new solutions. As we have seen in Section 2.2, the emerging inconsistencies not only indicated that there is evidence against the hypotheses (PEP) and (PDC), but their complementary nature provided a pattern that suggested (PDR). Fourth, while Baltin treats (IDR) only as a temporary hindrance that may be overcome later, (IEP1)-(IMD2) are regarded as strong counter-arguments against the hypothesis at issue which lead to the rejection of the hypothesis in question. Paraconsistent logic, however, does not allow us to compare the "weight" or "seriousness" of inconsistencies in such cases but allows us to reconstruct them as weak inconsistencies.

Therefore, it is not sufficient to assume mechanically that strong inconsistencies are destructive while weak ones are acceptable. The metascientific reconstruction should provide tools for deciding between alternative ways of splitting the theory into component possible worlds. A prerequisite of this is that we need criteria to judge whether the two possible worlds into which the theory at issue has been decomposed are well-motivated and may legitimise the permanent toleration of inconsistencies or ad hoc constructs which may be accepted only temporarily and by no means automatically, but only after a thorough examination of the whole situation. For example, a trivial ad hoc proposal, namely the wellknown strategy of labelling the 'disobedient' cases 'exceptions' can also be reconstructed, as we have seen in (20) and (21), with the help of weak inconsistency.

Accordingly, the mechanical application of paraconsistent logic would lead to an unrealistic methodology. Paraconsistent logic would enable us to legitimise considerations which are the results of the superposition of two possible worlds but which are clearly counter-intuitive, ad hoc or unpromising solutions from the linguist's object-theoretic point of view. Hence, there is a tension between two aspects of the paraconsistent metascientific reconstruction of inconsistency in linguistics. Paraconsistent logic is too strong to model inconsistency in linguistic theorising because it allows us to resolve many different kinds of contradictions by relying on the application of weak inconsistency. At the same time, paraconsistent logic is not strong enough to model inconsistency in syntactic theorising 
because it does not differentiate between well-motivated and ad hoc solutions and is not able to rank the rival proposals.

\subsection{Summary}

A workable metatheory of linguistic argumentation cannot dispense with paraconsistent tools which are necessary for the appropriate metascientific reconstruction of contradictory pairs of hypotheses in object-scientific theories. Paraconsistent logic provides the means to handle inconsistencies in cases in which both statements contradicting each other have to be retained in such a way that their maintenance does not lead to logical chaos. Our reconstruction of the inconsistencies in Baltin's approach, however, has pointed out that paraconsistent logic is not capable of mirroring the process of inconsistency resolution, the continuous emergence of new rival solutions and further inconsistencies, as well as the decision process between systems of hypotheses and inconsistencies. Therefore, in order to decide whether a particular treatment of a contradiction is possible, acceptable, preferable etc. or not, we have to supplement paraconsistent logic by further means. Accordingly, our findings are the starting point rather than the outcome of the process of raising the issue of inconsistency in syntactic argumentation. The reasons are as follows.

This metatheoretical model has to be capable of grasping, above all, two important, but so far unrevealed, characteristics of Baltin's argumentation. First, the choice of how to treat an inconsistency crucially depends on the comparison of the plausibility (reliability, acceptability) of the members of the given contradiction. Baltin never gives up "linguistic data", that is, hypotheses capturing generalisations about characteristics of sentences with a certain structure against proposals raised to solve (I); likewise, in most cases, he rejects the tested hypotheses instead of well-known hypotheses of generative grammar. Second, despite the central importance of such considerations, the plausibility of particular statements is only one of the factors which are taken into consideration when he chooses between rival alternatives, because he always compares whole solutions after investigating how problematic they are and how strong their explanatory power is. Baltin gives up a proposal only if there is another one which seems to be a better alternative.

From these findings it follows that the logical reconstruction of a given state of the theory is not enough to establish that it is consistent because during new stages of argumentation further contradictions may arise. Accordingly, problem solving is a continuously changing, dynamic process. Accordingly, due to this dynamism, the treatment of inconsistencies should be evaluated with respect to the whole process of theorising instead of being restricted to a particular stage of this process.

The above considerations make it clear that the evaluation of Baltin's approach is extraordinarily complex, because the choice between competing alternatives cannot be based solely on formal considerations, i.e., on the logical reconstruction of the theory. Therefore, although the scope of paraconsistent logic is much wider than that of classical logic, due to its substantially formal and static nature, not even paraconsistent logic can capture the nonformal and dynamic aspects of Baltin's argumentation. Paraconsistent logic is not capable of accounting for the heuristic function which inconsistencies play within scientific theories, has no tools for modelling the continuous emergence of inconsistencies during the process of scientific theorising, and cannot judge how strong or how weak the counter-arguments against the given theory-version which yield inconsistencies are. Paraconsistent logic is a useful tool for the metascientific reconstruction of certain kinds of inconsistency in a given state of the theorising process, but it is not able to capture and evaluate the different mechanisms of inconsistency-resolution. While the reconstruction of inconsistency "does fall 
within the province of logic, its resolution is inevitably an extralogical matter" (Rescher 1964: 37).

Consequently, one of the most stimulating but almost entirely unsolved tasks is to elaborate a metatheory of syntactic theorising which is able to account for the factors influencing the decision between different possible alternative treatments of inconsistencies.

At this point we risk the bold hypothesis that the metatheory we need can be constructed by supplementing paraconsistent logic with a model of plausible argumentation. We expect the latter to answer - at least partially - $(\mathrm{P})(\mathrm{c})$. The effectiveness of plausible argumentation is rooted in the circumstance that it is capable of integrating paraconsistent logic while it transgresses its limits by accounting for significant differences between alternative treatments of contradictions.

\section{4. $\quad$ Ad $(P)(c):$ Inconsistency and plausible argumentation 4.1. The $p$-model of plausible argumentation}

The basic idea of the p-model of plausible argumentation is that the structure of linguistic theories is based on various techniques of plausible argumentation. Such an approach has been put forward in Kertész \& Rákosi (2012). ${ }^{18}$ In the next paragraphs we briefly summarise those fundamental principles which are crucial for the understanding of our solution to $(\mathrm{P})(\mathrm{c})$; for their comprehensive discussion see Kertész \& Rákosi (2012).

(i) Plausible statements. In most cases our hypotheses are not statements the truth of which is guaranteed. Rather, they are more or less plausible statements: we are ready to accept them to some extent on the basis of sources that support them. ${ }^{19}$ Plausibility is gradual and strongly source-dependent. A statement may be very plausible according to one source, and less plausible with respect to other sources. It may also happen that some sources support the negation of the given statement and make it implausible. Further, if several sources support a statement, then its plausibility value is higher on the basis of all sources together than its plausibility value on the basis of any of the sources alone. It is also possible that neither a statement nor its negation is made plausible by a source included in the p-context; in such cases, we speak of neutral plausibility on the basis of the given source.

For example, in Baltin (1987), the statement 'The sentence People were so angry who knew John that they refused to participate. is grammatically correct and contains a relative clause and a degree word complement clause' is plausible on the basis of Baltin's linguistic intuition as a source. It is not true with certainty, because introspection cannot be regarded as a completely reliable source (cf. e.g. Schütze 1996, Featherston 2009) in linguistics. Similarly, the hypothesis 'Elements moving rightward cannot be moved out of the clause in which they originate' (cf. (IEP1)(a)) is a plausible statement among others on the basis of a linguistic paper (Ross 1967) as a source, because it is expressly supported by it. Despite this, the paper cited cannot be regarded as a perfectly reliable source guaranteeing the truth of this statement.

Plausibility can be represented numerically. These values merely indicate different relative strengths of reliability, supportedness, acceptance within a theory (i.e., argumentation process). Therefore, their manipulation cannot be described with the help of a logical calculus, it is more rudimentary. The plausibility value of statement $p$ on the basis of the

\footnotetext{
18 Our approach has been motivated by the ideas of George Polya and Nicholas Rescher (cf. e.g. Polya 1948, 1954; Rescher 1976, 1987), and prepared e.g. in Kertész (2004) and Kertész \& Rákosi (2006, 2009).

19 Such - more or less reliable - sources are in linguistics, for example, corpora, theories, conjectures, the intuition of native speakers, experiments, fieldwork, historical documents, dictionaries, videotapes, or even inferences (cf. Rescher 1976: 6f.; Rescher 1973: 63ff., Kertész \& Rákosi 2012: 63ff.).
} 
source $S$ is such that:

$|p|_{S}=1$, if $p$ is true with certainty on the basis of $S$;

$|p|_{S}=0$, if $p$ is of neutral plausibility on the basis of $S$;

$0<|p|_{S}<1$, if $p$ is plausible on the basis of $S$.

(ii) Plausible inferences. Deductive inferences the premises of which are true with certainty secure the truth of the conclusion. However, very often either there is no logical consequence relation between the premises and the conclusion or at least one of the premises is, instead of being certainly true, only plausible in the light of the given sources. Moreover, the combination of these two cases may also occur. In such situations, we obtain plausible inferences that are capable of making their conclusion plausible - but not true - on the basis of the content and plausibility value of their premises. Moreover, if there is no logical consequence relation between the premises and the conclusion, then latent background assumptions are also needed which have to be plausible or at least not known to be implausible or false with certainty. In such inferences the connection between the premises and the conclusion cannot be reduced to the relationship between their logical structures but always rests on a semantic relation such as causality, analogy, similarity, sign, necessary or sufficient condition, part-whole relation etc.

For example, Baltin's argumentation concerning the use of (4) as evidence for (PEP) in Section 2.2 can be reconstructed as follows:

$0<\mid$ If (PEP) and relative clauses are adjoined to S, then the sentence People were so angry who knew John that they refused to participate is grammatically correct, while the sentence People were so angry that they refused to participate who knew John is ungrammatical. $\left.\right|_{S I}<1$

$0<\mid$ Relative clauses are adjoined to $\mathrm{S} .\left.\right|_{S 2}<1$

$0<\mid$ The sentence People were so angry who knew John that they refused to participate is grammatically correct, while the sentence People were so angry that they refused to participate who knew John is ungrammatical. $\left.\right|_{S 3}<1$

$0<|(\mathrm{PEP})|_{I}<1$

It is easy to see that there is no logical consequence relation between the premises and the conclusion. Despite this, the latter can be deemed plausible because all premises are plausible according to some source (linguistic intuition, linguistic analysis, Generative Grammar, etc.), and this inference can be extended by plausible latent background assumptions and transformed into a deductively valid inference. Such a latent background assumption is, for example, that in all other sentences containing a relative clause and a complement clause, the former precedes the latter, too.

There are also plausible inferences which are deductively valid but whose premises are only plausible instead of being true with certainty. Thus, they cannot make their conclusion true with certainty but they can make them plausible:

$0<\mid$ If (PEP), then every sentential complement should be associated with exactly one degree word. $\left.\right|_{S I}<1$

$0<\mid$ If every sentential complement should be associated with exactly one degree word, then the sentence So many people have read so many books so often that it's hard to keep up with them is ungrammatical. $\left.\right|_{S 2}<1$

$0<\mid$ The sentence So many people have read so many books so often that it's hard to keep up with them is grammatically correct. $\left.\right|_{S 3}<1$ 


$$
\left.0<|\sim(\mathrm{PEP})|_{I}<1\right)
$$

This pair of inferences, which we reconstructed above, is typical of linguistics, because in most cases we have to face situations in which there are pieces of evidence which support (but do not prove conclusively) a hypothesis while other pieces of evidence provide counterarguments against it, without showing it to be false with certainty.

(iii) The p-context. Besides the logical analysis of the premises and the conclusion, the creation, usage and evaluation of plausible inferences also have to rely on all information that may be relevant for judging the plausibility value of the premises and the semantic relation between them and the conclusion. Therefore, we introduce the concept of the p-context. The p-context includes the sources in terms of which the plausibility value of statements can be judged as well as a set of statements together with their relevant characteristics such as their plausibility values with respect to the sources in the p-context and their logical and semantic structure. The methodological tools related to the components of the p-context (for instance, the strategies of the resolution of inconsistencies, the methods for judging the reliability of the sources etc.) and the criteria of their usage also belong to the p-context.

(iv) The p-context-dependency of plausible inferences. Plausible inferences are in several respects p-context-dependent. First, the premises have to be plausible or true according to some sources in the p-context. Second, new sources may become available which make the premises more plausible or less plausible or even implausible. In such cases, the plausibility of the conclusion will change as well. Third, if there is no logical consequence relation between the premises and the conclusion, then latent background assumptions are also needed (Rescher 1976: 60ff., Polya 1948: 223). These background assumptions have to possess certain logical and semantic characteristics (see Kertész \& Rákosi 2012: 88ff.), and they have to be true, plausible, or at least not implausible or false according to some source in the p-context.

(v) The heuristic function of plausible inferences. Plausible inferences are effective heuristic tools. First, they can be applied in p-contexts in which inferences that could secure the truth of the conclusion are not available, giving it a partial support. Second, plausible inferences having latent background assumptions also provide new pieces of information not even implicitly included in the premises because their conclusion will contain the information content of the latent background assumptions as well (Polya 1948: 221; Rescher 1976: 60ff., 97ff.).

(vi) The informational over- and underdetermination of the p-context. In a p-context the sources may yield too much information in the sense that there is a statement which is made plausible by some source while its negation is made plausible by another. In such cases, the p-context is informationally overdetermined (Rescher 1976: 2, Rescher \& Brandom 1980: $3 \mathrm{ff}$.) and the set of the plausible statements in the p-context is p-inconsistent. Nevertheless, the p-context may be informationally underdetermined as well (Rescher \& Brandom 1980: $3 \mathrm{ff}$.). A typical case of the informational underdetermination of the p-context is its $p$ incompleteness, insofar as there are statements which are neither plausible (in the extreme case: true with certainty) nor implausible (in the extreme case: false with certainty) with respect to any source given. A p-context may be simultaneously informationally under- and overdetermined.

(vii) Problems, their solution and their resolution. We call instances of informational over- or underdetermination p-problems. If a p-context is characterised by over- and/or underdetermination, then it is called p-problematic. In order to solve a problem, we have to re-evaluate the p-context by revising its elements (see (viii) on this). A solution of a $p$ problem is achieved if a p-context has been arrived at in which either (a) the statement in question is unanimously supported or opposed by the sources, that is, it is either plausible or 
implausible on the basis of all sources in the given p-context, or (b) the statements generating p-inconsistency can be represented in such a way that they become separated systematically and this separation is well-motivated.

It is possible, however, that a problem has several solutions. The resolution of a $p$ problem is a solution of the given problem which is, when compared with other solutions, the best according to a set of criteria accepted and according to the information available to us.

(viii) Plausible argumentation. 'To achieve the solutions or the resolution of a given $\mathrm{p}$ problem, we need a heuristic tool that enables us to re-evaluate the p-context. This heuristic tool is what we will call plausible argumentation. In simple terms, plausible argumentation is the transformation of a p-problematic p-context into one which is no longer (or at least, less) problematic. This involves the successive re-evaluation of a problematic $p$-context by the elaboration of possible solutions to its p-problems, the evaluation of the alternative solutions and the comparison of the latter. Its aim is the detection of all available solutions and the decision as to which of them is to be accepted as the resolution of the given p-problem.

(ix) The cyclic nature of plausible argumentation. The above characterisation of plausible argumentation indicates that the argumentation process is basically not linear, because the re-evaluation of a problematic p-context usually does not lead immediately to an unproblematic one but may raise new problems. This may require the revision of previous decisions, the assessment of other alternatives etc. Therefore, throughout the argumentation process one returns to the problems at issue again and again, and re-evaluates the earlier decisions about the acceptance or rejection of statements, the reliability of the sources, the plausibility values of the statements, the workability of methodological norms, the conclusions previously reached by inferences etc. In sum: one retrospectively re-evaluates the information at one's disposal (cf. Rescher 1976, 1987). Thus, retrospective re-evaluation is cyclic in nature.

An argumentation cycle is a phase of the plausible argumentation process which develops a new solution to the p-problems contained in the starting p-context and examines whether with this solution their resolution has been achieved. It may have sub-cycles which revise certain decisions made within the given argumentation cycle, and continue the argumentation process by turning back to an earlier stage of the argumentation cycle. It consists of three main stages.

First, one has to set up a new p-context version. One presents hypotheses, sources, and methodological norms which one accepts and which are regarded as the starting points of the next stage (cycle) of the argumentation process. The new p-context version may contain the starting p-problem (that is, it may cover both members of a p-inconsistency or remain $p$ incomplete), ${ }^{20}$ it may be developed around one of the rival hypotheses by omitting the other one, it may be the continuation of an earlier p-context variant (that is, the argumentation process may turn back to an earlier stage), or it may combine and develop two alternative pcontext versions in parallel but systematically separated.

Second, the new p-context version has to be elaborated. With this aim in view, one has to search for pieces of information from the sources in the p-context that seem to be relevant to the solution of the starting p-problem(s). That is, the p-context has to be extended in such a way that one assigns a plausibility value to further statements with the help of sources not used so far and by drawing new inferences, or one applies methods not yet used, or makes use of new criteria, etc. The information obtained has to be systematised; that is, the extended p-context has to be coordinated: the plausibility values of statements stemming from the old and the new sources, their relevant characteristics, as well as old and new pieces of information related to the reliability of the sources have to be compared. Then, the coordinated context 
has to be modified; one has to work out the p-context that will be regarded as the revised, reevaluated version of the starting p-context, and might provide a solution of the starting pproblem. For example, one has to determine the plausibility value of the statements on the basis of the sources at one's disposal; it has to be decided which sources should be considered unreliable, and the information originating from them has to be omitted; certain methodological norms have to be abandoned or modified or new ones have to be accepted; on the basis of the latter, further sources can be deemed reliable and may be used etc.

Third, one has to compare the elaborated p-context version to its rivals: is it a better solution of the starting p-problem, is it a resolution (that is, the best solution available) or not? If it is not better than the solutions obtained in the earlier cycles or it is not yet a solution, then one has to turn back to this cycle and further elaborate it by starting a new subcycle. However, in most cases the choice among the rival solutions cannot be definite and comprehensive but remains partial and heuristic. This is due to the complexity and the diversity of the relevant factors which might have an impact on the decision on the one hand and to the openness of the argumentation process on the other hand - that is, in possession of new information, earlier decisions can always be re-evaluated. For further details, see Kertész \& Rákosi (2012: 153ff.).

Figure 6 illustrates how the central concepts of plausible argumentation are related: 


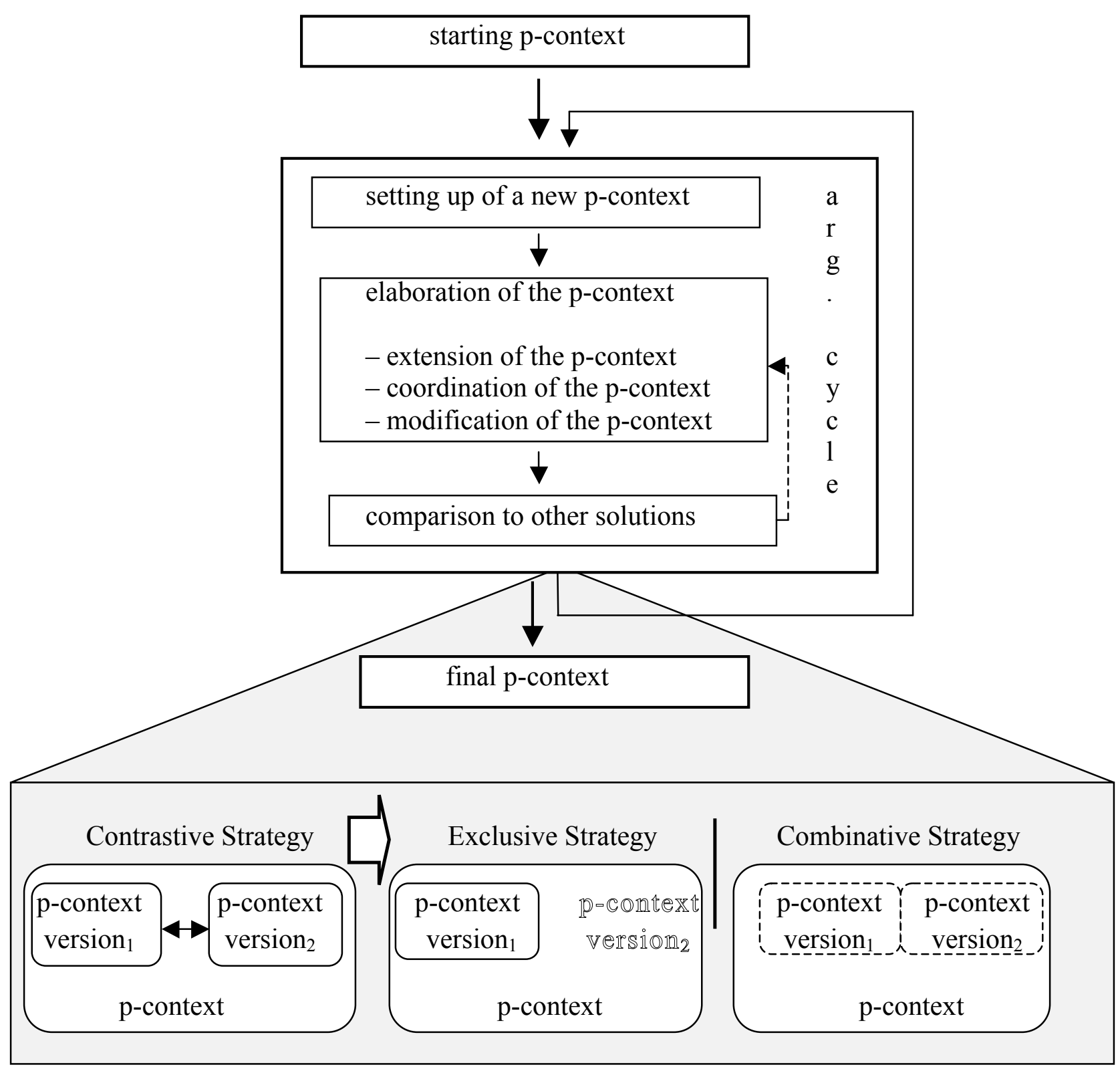

Figure 6

(x) The prismatic nature of plausible argumentation. Plausible argumentation is not only cyclic, it is also prismatic. This means that the cycles continuously change the perspective from which the pieces of information constituting the p-context are evaluated (cf. Rescher 1987). The "prism" through which, during the argumentation cycles, one re-evaluates the information at one's disposal and/or views new pieces of information to be considered, has different sides which do not follow prefabricated patterns.

In this way, one modifies the p-context again and again and - if no resolution of the starting p-problem is achieved - sets up new, re-evaluated versions of it. Thus, the newer and newer argumentation cycles gradually elaborate diverse constellations of statements, plausibility values, sources, and methodological principles etc., that is, rival solutions. Of course, cyclic returns are also possible within an argumentation cycle. If one is not satisfied with the fulfilment of some step during the current cycle in the face of the information obtained in a later stage, then one may start a sub-cycle.

A constitutive characteristic of plausible argumentation is that usually the re-evaluation 
of the p-context cannot be complete. There are often practical limits that do not allow us, for example, to take every source of the starting p-context into consideration, to produce and evaluate all information potentially inherited by them, or to perform a complete test of inconsistency during the coordination of the p-context etc. During the cyclic returns, one cannot elaborate and compare every possible solution or solution-candidate. Therefore, one has to rely on problem-solving strategies that are capable of ensuring that the argumentation process takes a relatively comprehensive range of information into consideration. On the basis of the partial accomplishment of the steps in Figure 6, such heuristics enable us to arrive at a fair number of solutions to the p-problems investigated, and via this, they may lead to reliable resolutions of the p-problems within the given practical limits.

The choice of the problem-solving strategy which can be applied in the current state of the elaboration of the p-context requires the use of heuristic considerations as well, because there are no exact criteria which would allow for determining which perspective has to be taken in a given situation. Basically, one can choose between three strategies of treating $\mathrm{p}$ inconsistency:

- The Contrastive Strategy. The essence of this strategy is that it develops p-context version germs in such a way that it treats the p-context versions containing the contradictory statements as rival alternatives. That is, one aims at reaching a decision between them on the basis of the information at one's disposal.

- $\quad$ The Exclusive Strategy. This strategy is applied after the Contrastive Strategy when a decision has been reached between the two rival p-context versions elaborated. It aims at checking as comprehensively as possible whether the p-context version chosen can be extended in such a way that it is capable of explaining phenomena that could be accounted for within the rejected p-context version. This, of course, may lead to the emergence of new p-problems.

- The Combinative Strategy. This strategy consists in treating the two rival p-context versions not as rivals but as two parts of a whole which have to be maintained simultaneously. The two co-existing p-context versions making up the whole p-context have to be gradually elaborated. In such cases, one has to try to elaborate two unproblematic p-context versions. If one succeeds, then the starting p-problem is solved by the simultaneous but systematically separated maintenance of the two rival pcontext versions. Nevertheless, it is essential to check whether the separation of the two p-context versions is well-motivated within the whole p-context.

\subsection{Beyond the limits of paraconsistent reconstruction}

Basically, there are three groups of hypotheses in Baltin's argumentation:

- "Linguistic data", that is, hypotheses capturing characteristics of sentences with a certain structure. Baltin never gives them up when they are in conflict with other hypotheses. These statements result from the linguistic analysis of sentences and capture generalisations. Therefore, they cannot be considered certainly true, but have to be assigned a very high plausibility value.

- Well-established, generally accepted hypotheses of generative grammar. Since they have already been applied successfully to several fields of linguistic analysis, their abandonment would have profound consequences for generative grammar. Thus, they receive a high plausibility value.

- $\quad$ Hypotheses put forward to treat (I). They are regarded as conjectures supported only 
to a certain extent and which are related only to a subpart of the theory, that is, to the description of the structure of sentences containing degree words and their sentential complements. Therefore, they have only a relatively low plausibility value.

The assignment of these plausibility values is based on the reliability of the source of the plausible statement at issue. These initial values may, however, change during the argumentation process.

The starting p-context is p-inconsistent because (I)(a) and (b) are plausible according to some sources but they contradict each other. Baltin's argumentation aiming at the resolution of (I) can be divided into 4 stages. Each stage can be interpreted as an argumentation cycle, in which rival hypotheses about the solution of (I) are put forward and evaluated.

In Cycle 1, Baltin constructs a p-context around (PEP) as a possible solution to the problem (I). Relying on the results of our paraconsistent reconstruction it is easy to see that this solution is based on the Combinative Strategy: (I)(a) and (I)(b) are maintained simultaneously as belonging to two different p-context-versions, to the set of hypotheses describing the deep structure and to the set of hypotheses related to the surface structure of English sentences, respectively. Then, the plausibility of (PEP) is tested with the help of (4)(8). Arguments which make use of (4) and (5) increase the plausibility value of (PEP). For example, with the help of (4) one obtains the following plausible inference: ${ }^{21}$

$0<\mid$ If (PEP) and relative clauses are adjoined to $\mathrm{S}$, then the sentence People were so angry who knew John that they refused to participate is grammatically correct, while the sentence People were so angry that they refused to participate who knew John is ungrammatical. $\left.\right|_{S I}<1$

$0<\mid$ Relative clauses are adjoined to $\mathrm{S} .\left.\right|_{S 2}<1$

$0<\mid$ The sentence People were so angry who knew John that they refused to participate is grammatically correct, while the sentence People were so angry that they refused to participate who knew John is ungrammatical. $\left.\right|_{S 3}<1$

$0<|(\mathrm{PEP})|_{I}<1$

The first premise can be regarded as plausible on the basis of theoretical considerations as we have seen in Section 2.1. The second premise has a moderate plausibility value, while Baltin assigns a high plausibility value to the third premise. We have shown in Section 4.1 (ii) that, although this inference is not deductively valid, it is capable of making its conclusion plausible. Thus, the initial plausibility of (PEP) which it gained as a conjecture has increased. The application of (5) leads to similar results; thus, (PEP) has become more plausible. (6)-(8) and the conflict between (PEP) and Ross's Right Roof Constraint (cf. (IEP1)-(IEP4)), however, point in the opposite direction: the plausible inferences which contain them as premises support the implausibility of (PEP). For example, in connection with (7) the following plausible inference presents itself:

$0<\mid$ If (PEP) and the Condition on Extraction Domains hold, then Extraction is not allowed from the sentential complement of degree words. $\left.\right|_{\mathrm{B}}<1$

$0<\mid$ If Extraction is not allowed from the sentential complement of degree words, then the sentence Who was he too angry to visit ? is ungrammatical. $\left.\right|_{\mathrm{B}}<1$

$0<\mid$ The sentence Who was he too angry to visit __ ? is grammatical. $\left.\right|_{\mathrm{B}}<1$

$0<\mid$ The Condition on Extraction Domains hold. $\overline{\left.\right|_{\mathrm{B}}<1}$

$210<|\mathrm{p}|_{\mathrm{S}}<1$ indicates that statement $p$ is plausible on the basis of source $S$; here, $B$ signifies Baltin (1987) as a direct source, $I$ the given inference as an indirect source; for the concepts of direct/indirect source, see Kertész \& Rákosi: 70ff.). 


$$
0<|\sim(\mathrm{PEP})|_{\mathrm{I}}<1
$$

This inference makes the negation of (PEP) plausible - that is, it leads to p-inconsistency. The structure of the p-context after its extension with the data and inferences mentioned and their coordination is illustrated in Figure 7.

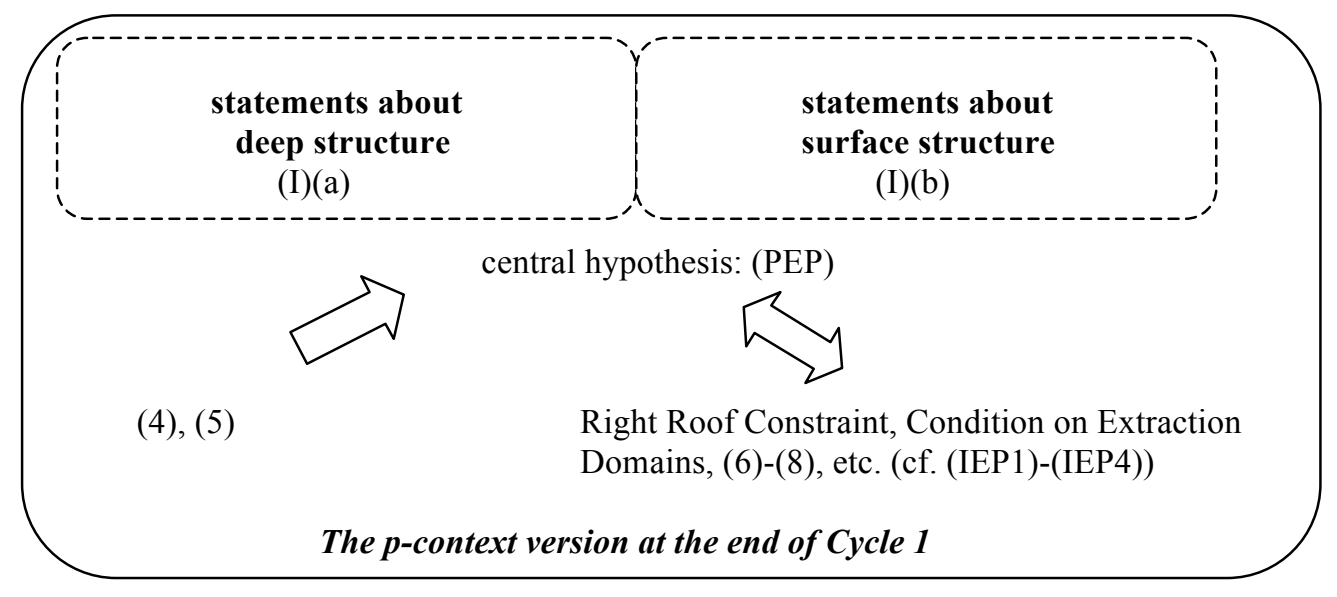

Figure $7^{22}$

The plausibility of the negation of (PEP) is higher than the plausibility of (PEP) on the basis of the sources taken into consideration, and the maintenance of (PEP) would boil down to the tolerance of four inconsistencies which can be represented as ad hoc weak inconsistencies (cf. (IEP1)-(IEP4)). Therefore, Baltin gives up (PEP) and examines a rival hypothesis in Cycle 2.

In Cycle 2, Baltin builds the second p-context version around the hypothesis (PDC). (PDC) is based on the application of the Combinative Strategy, too, because it retains and separates (I)(a) and (b). (PDC)'s initial plausibility is increased by inferences relying on (6)(8) and Ross's Right Roof Constraint, but its negation becomes plausible as a consequence of setting up inferences with the help of (4) and (9) (cf. (IDC1)-(IDC2)). Figure 8 illustrates the p-context version arrived at in Cycle 2 .

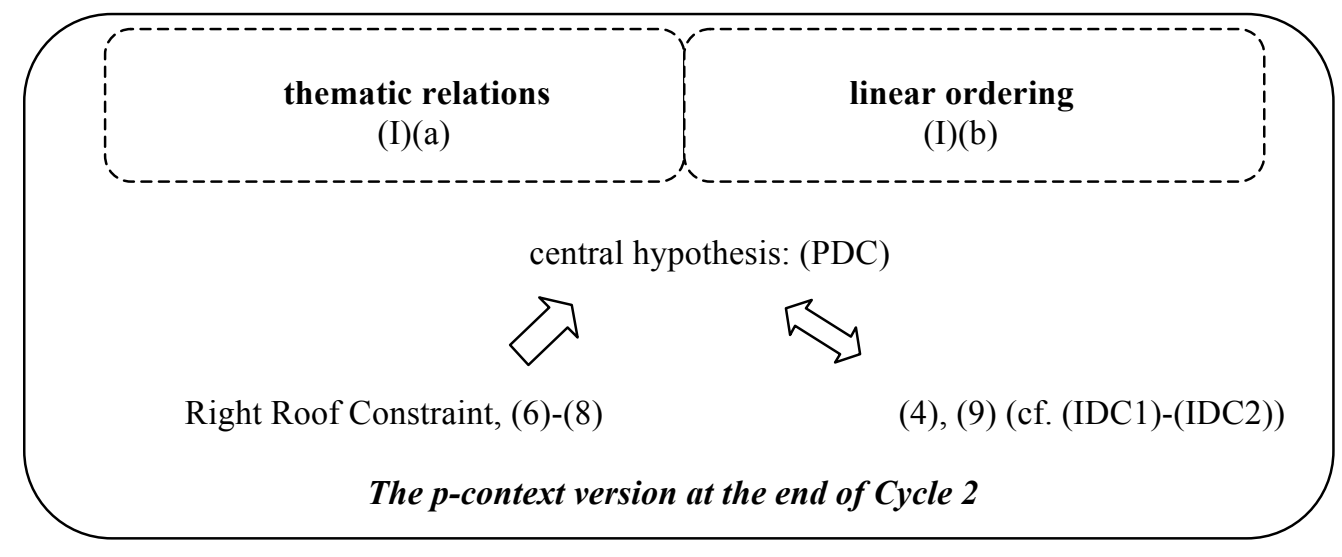

Figure 8

Since (PDC) can be accepted only if one tolerates two inconsistencies which can be represented as ad hoc weak inconsistencies (cf. (IDC1)-(IDC2)), Baltin raises a third rival

22 Simple thick arrows indicate weak evidence for the central hypothesis of the given p-context version, while double thick arrows stand for weak evidence against it. 
hypothesis.

So far Baltin has taken the Contrastive Strategy. In Cycle 3, however, he does not treat (PEP) and (PDC) as rivals but maintains the structures resulting from their application simultaneously, without separating them. Therefore, it is not only the case that the explanatory power of one hypothesis and its rival are added but all counter-examples remain effective, as (IMD1) witnesses. This means that this p-context version is clearly untenable, for it leads to logical chaos as illustrated in Figure 9.

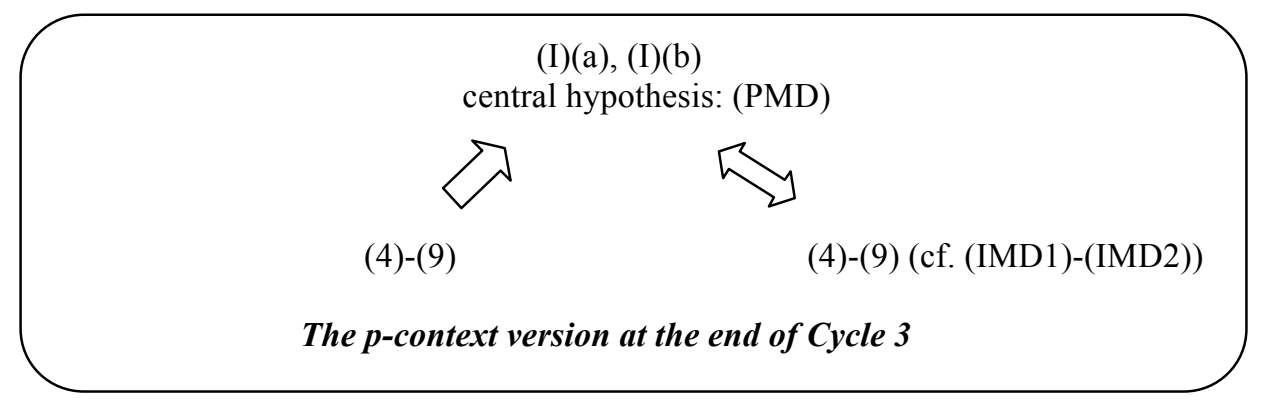

Figure 9

As Figure 10 shows, in Cycle 4 Baltin draws the moral that the two structure-descriptions have to be separated and raises (PDR) - that is, he makes us of the Combinative Strategy.

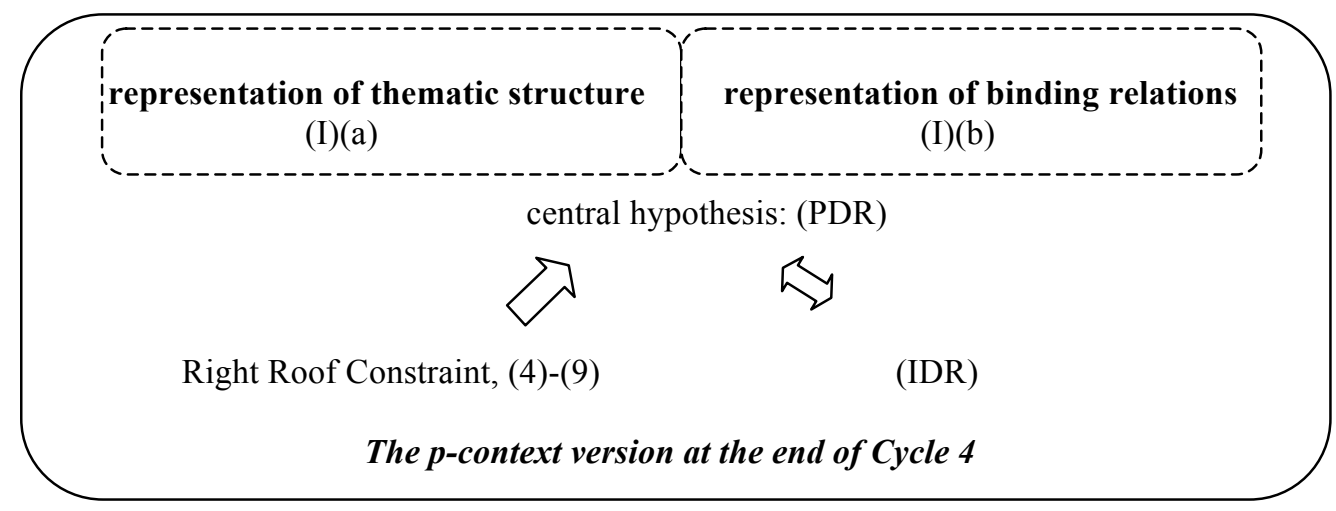

Figure 10

In this case, the plausibility of (PDR) is definitely higher than that of its negation on the basis of the sources in the p-context. Moreover, (IDR) can be regarded as an ad hoc weak inconsistency in which case there is real hope that in the future it can be re-interpreted in a non-ad hoc way. Namely, it may turn out at a later stage of the development of generative linguistics that there is some principled reason why degree word complement clauses (and, possibly, other structures as well) require double representation while most structures do not. Then, (IDR) could be resolved in that the p-context is completed by further hypotheses which would allow for a well-founded separation of the p-context into two p-contexts and the application of the Combinative Strategy.

By taking an evaluative perspective, Baltin compares the four p-context versions (rival solutions) and chooses the fourth, because it can be regarded as the best solution (resolution) of the starting inconsistency on the basis of the sources taken into account in Baltin (1987).

It may happen, however, that new inconsistencies emerge which increase the implausibility of (PDR) and point to another alternative solution. For example, White (1997) and Larson (1988) claim that negative polarity items and sentences containing double object verbs are counter-examples against (PDR). A third type of inconsistency emerges from the 
fact that the hypothesis of the double representation strongly contradicts the principles of generative grammar.

\subsection{Summary}

The paraconsistent representation of Baltin's account could easily be integrated with the pmodel of plausible argumentation. First, paraconsistent logic is the logical basis of the Combinative Strategy. Second, since the p-model interprets linguistic theorising as a problem solving process, it is capable of accounting for the emergence and function of inconsistencies in linguistic theorising. Third, it makes it possible to differentiate between ad hoc weak inconsistencies which may be only temporarily tolerable, and well-motivated ones in which the two members of the inconsistency are systematically separated and can be tolerated permanently. Fourth, the p-model of linguistic argumentation evaluates inconsistencies not in isolation but as part of the p-context which covers all information available and as arguments which play a role in the decision among rival p-context versions.

\section{Conclusions}

According to the standard view of the analytical philosophy of science, inconsistencies force the scholar to give up her theory because it has been falsified, compelling her to elaborate a new theory in which this contradiction does not appear. In this view, inconsistencies cannot be tolerated within theories; they can perform their heuristic function outside theories, that is, by forcing scientists to develop new ones. As we have seen, however, there is a wide gap between these norms and linguists' practice. The radical consequence of these considerations is, of course, that the metatheory that underlies the clash between the methodological prejudices of mainstream linguistics and the practice of linguistic research has to be discarded and another one has to be chosen.

Now the question was, of course, what kind of new metatheory one should vote for. At first sight, paraconsistent logic seemed to provide the means to handle inconsistencies in cases in which both contradictory propositions can be retained in such a way that their maintenance does not lead to logical chaos. Our analysis of Baltin's argumentation casts light upon the finding that weak inconsistency and its paraconsistent treatment is by no means something exotic. On the contrary: paraconsistency is quite a natural everyday practice in syntactic argumentation.

However, the static nature of paraconsistent logics does not permit us to reconstruct the process of the continuous emergence and resolution of inconsistencies, and it does not provide tools for grasping the different strategies in treating and comparing the possible alternatives. Paraconsistent logic is a formal system which can only account for a momentary state of the process of scientific theorising. It is not able to grasp the development and dynamics of the process of theory formation, that is, it has no means for reconstructing the relations among the different stages of inconsistency-resolution. Therefore, although paraconsistent logic proved to be an indispensable and extremely fruitful tool for the metascientific reconstruction of inconsistencies, it has its limits and needs to be refined because it can provide us only with a partial methodology. It can be, however, easily integrated with the p-model of plausible argumentation which might yield a complex, workable and heuristically fertile methodology. 


\section{Acknowledgements}

Work on the present paper was supported by the MTA-DE Research Group for Theoretical Linguistics and the project OTKA K 77823. Csilla Rákosi's work was also supported by the János Bolyai Research Scholarship of the Hungarian Academy of Sciences.

\section{References}

Baltin, M.R. (1987). Degree complements. (In G.J. Huck, \& A.E. Ojeda (Eds.), Discontinuous constituency (pp. 11-26.) Orlando: Academic Press.)

Batens, D. (2001). A General Characterization of Adaptive Logics. Logique et Analyse, 173175: 45-68.

Batens, D. (2007). A Universal Logic Approach to Adaptive Logics. Logica Universalis, 1: 221-242.

Carnielli, W.A., Farinas del Cerro, L. \& Lima Marques, M. (1991). Contextual negations and reasoning with contradictions. (In 12th International Joint Conference on Artificial Intelligence), (pp. 532-537) (Sydney: Morgan Kaufman).

Carnielli, W.A., M.E. Coniglio \& J. Marcos (2007). Logics of Formal Inconsistency. (In D. Gabbay \& F. Guenthner (Eds.), Handbook of Philosophical Logic Volume 14 (Second Edition (pp. 15-107.) Berlin: Springer).

Chomsky, N. (1980). On binding. Linguistic Inquiry, 11, 1-46.

Chomsky, N. (2002). On nature and language (Cambridge: Cambridge University Press).

da Costa, N.C.A. (1974). On the Theory of Inconsistent Formal Systems. Notre Dame Journal of Formal Logic, 15 (4): 497-510.

Featherston, S. (2009). Relax, lean back, and be a linguist. Zeitschrift für Sprachwissenschaft $28,127-132$.

Guéron, J. \& May, R. (1984). Extraposition and logical form. Linguistic Inquiry, 15, 1-31.

Jaśkowski, S. (1969 [1948]). Propositional Calculus for Contradictory Deductive Systems, Studia Logica, 24: 143-157.

Kertész, A. (2004). Philosophie der Linguistik. Studien zur naturalisierten Wissenschaftstheorie (Tübingen: Narr).

Kertész, A. (2012). The ,Galilean Style in Science' and the Inconsistency of Linguistic Theorising. Foundations of Science 17, 91-108.

Kertész, A. \& Rákosi, Cs. (2006). Inconsistency and plausible reasoning in an analysis of German affricates. A case study in the philosophy of linguistics. Language Sciences, 28, 386-423.

Kertész, A. \& Rákosi, Cs. (2009). Cyclic vs. circular argumentation in the Conceptual Metaphor Theory. Cognitive Linguistics, 20, 703-732.

Kertész, A. \& Rákosi, Cs. (2012). Data and evidence in linguistics. A plausible argumentation model (Cambridge: Cambridge University Press).

Larson, R.K. (1988). On the double object construction. Linguistic Inquiry, 19, 335-391.

Meheus, J. (Ed.)(2002). Inconsistency in science (Dordrecht: Kluwer).

Meheus, J. (2002): Preface. In J. Meheus (Ed.), pp. vii-ix.

Moravcsik, E. (2006). An introduction to syntactic theory. (London \& New York: Continuum).

Moravcsik, E. (2010). Conflict resolution in syntactic theory. Studies in Language, 34, 636669.

Nickles, T. (2002). From Copernicus to Ptolemy. Inconsistency and method. (In J. Meheus (Ed.) (pp. 1-33)). 
Polya, G. (1948). How to solve it (Princeton: Princeton University Press).

Polya, G. (1954). Patterns of plausible inference (London: Oxford University Press).

Priest, G. (2001). Paraconsistent belief revision. Theoria, 67/3, 214-228.

Priest, G. (2002a). Inconsistency and the empirical sciences (In J. Meheus (Ed.), (pp. 119128)).

Priest, G. (2002b): Paraconsistent logic. (In D. Gabbay \& F. Guenthner (Eds.), (pp. 287-393), Handbook of paraconsistent logic Dordrecht: Kluwer).

Priest, G., Beall, J.C. \& Armour-Garb, B. (eds.)(2004). The law of non-contradiction (Oxford: Clarendon Press).

Priest, G. \& Tanaka, K. (2009): Paraconsistent logic. (In The Stanford Encyclopedia of Philosophy. http://plato.stanford.edu/entries/logic-paraconsistent/)(accessed 04. 09. 2012).

Rescher, N. (1964). Hypothetical reasoning (Amsterdam: North-Holland).

Rescher, N. (1973). The coherence theory of truth (Oxford: Clarendon Press).

Rescher, N. (1976). Plausible reasoning (Assen \& Amsterdam: Van Gorcum).

Rescher, N. (1987). How serious a fallacy is inconsistency? Argumentation, 1, 303-316.

Rescher, N. \& Brandom, R. (1980). The logic of inconsistency (Oxford: Blackwell).

Ross, J.R. (1967). Constraints on variables in syntax (Indiana University Linguistics Club, Bloomington).

Schütze, C.T. (1996). The empirical base of linguistics. Grammaticality judgments and linguistic methodology. (Chicago \& London: The University of Chicago Press).

Schotch, P.K. and R.E. Jennings (1980). Inference and Necessity, Journal of Philosophical Logic, 9, 327-340.

Tanaka, K., Berto, F., Mares, E. \& Paoli, F. (2013): Paraconsistency: Introduction. (In K. Tanaka, F. Berto, E. Mares \& F. Paoli (Eds.), (pp. 1-12), Paraconsistency: Logic and Applications, Dordrecht, Heidelberg, London, New York: Springer).

White, J.R. (1997). Result clauses and the structure of degree phrases. UCL Working Papers in Linguistics, 9: 1-20. 